\title{
VdNPS, a Nonribosomal Peptide Synthetase, Is Involved in Regulating Virulence in Verticillium dahliae
}

\author{
Xiumei Luo, $, 1,2, \dagger$ Tingting Tian, ${ }^{1}$ Xue Tan, ${ }^{1}$ Yixuan Zheng, ${ }^{2}$ Chengjian Xie, ${ }^{2} \mathrm{Ya} \mathrm{Xu},{ }^{2}$ and Xingyong Yang $2, \dagger$ \\ 1 The School of Life Science, Chongqing University, Chongqing 401331, China \\ ${ }^{2}$ Chongqing Engineering Research Center of Specialty Crop Resources and The College of Life Science, Chongqing Normal University, \\ Chongqing 401331, China \\ Accepted for publication 27 March 2020.
}

\begin{abstract}
Nonribosomal peptide synthetases (NPS) are known for the biosynthesis of antibiotics, toxins, and siderophore production. They are also a virulence determinant in different phytopathogens. However, until now, the functional characterization of NPS in Verticillium dahliae has not been reported. Deletion of the NPS gene in $V$. dahliae led to the decrease of conidia, microsclerotia, and pathogenicity. $\triangle V d N P S$ strains were tolerant to $\mathrm{H}_{2} \mathrm{O}_{2}$, and the genes involved in $\mathrm{H}_{2} \mathrm{O}_{2}$ detoxification, iron/copper transport, and cytoskeleton were differentially expressed in $\triangle V d N P S$. Interestingly, $\triangle V d N P S$ strains exhibited hypersensitivity to salicylic acid (SA), and the genes involved in SA hydroxylation were up-regulated in $\triangle V d N P S$ compared with wild-type
\end{abstract}

ABSTRACT
$V$. dahliae under SA stress. Additionally, during infection, $\triangle V d N P S$ induced more pathogenesis-related gene expression, higher reactive oxygen species production, and stronger SA-mediated signaling transduction in host to overcome pathogen. Uncovering the function of VdNPS in pathogenicity could provide a reliable theoretical basis for the development of cultivars with durable resistance against $V$. dahliaeassociated diseases.

Keywords: analytical and theoretical plant pathology, host response, nonribosomal peptide synthetase, reactive oxygen species, salicylic acid, vegetative growth, Verticillium dahliae, virulence
Nonribosomal peptides (NRPs) are an important group of natural products that exhibit a wide range of biological and pharmacological activities in medicine and agriculture, such as daptomycin, penicillin, and vancomycin (Sieber and Marahiel 2005). $\delta$-(L$\alpha$-aminoadipyl)-L-cysteinyl-D-valine is the firstly identified NRP in biosynthetic pathways of $\beta$-lactam antibiotics 40 years ago, of which penicillin and cephalosporin are the most well-known (Byford et al. 1997). Fungal toxins are a category of NRPs, including the HC-toxin produced by race 1 of the maize pathogen Cochliobolus carbonum (Panaccione et al. 1992), AM-toxin produced by the apple pathotype Alternaria alternata (Johnson et al. 2000), and enniatin produced by Fusarium spp. (Haese et al. 1993). These products are assembled by NRP synthetases (NPSs) that consist of a series of catalytic domains without the involvement of ribosomes (Fischbach and Walsh 2010). NPSs not only catalyze the synthesis of secondary metabolites but also determine virulence and iron metabolism. For example, AaNPS6 is involved in maintaining iron homeostasis via siderophore iron uptake and is a conserved virulence determinant through resisting to toxic reactive oxygen species (ROS) in the citrus fungal pathogen, Alternaria alternata (Chen et al. 2013), as well as in plant pathogenic ascomycetes, such as $C$. heterostrophus, C. miyabeanus, F. graminearum, and Alternaria brassicicola (Oide et al. 2006).

†Corresponding authors: X. Luo; luoxm@cqu.edu.cn, and X. Yang; yangxy94@swu.edu.cn

Funding: This work is founded by the Basic Research and Frontier Exploration Foundation of Chongqing (cstc2018jcyjAX0707 and cstc2018jcyjAX0753), National Key R\&D Program of China (2017YFE0115500), the National Natural Science Foundation of China (31801911), and China Postdoctoral Science Foundation (2017M622973).

*The $\boldsymbol{e}$-Xtra logo stands for "electronic extra" and indicates that supplementary material and supplementary tables are published online.

The author(s) declare no conflict of interest.

(c) 2020 The American Phytopathological Society
Deletion of NPS6 orthologs results in reduced virulence and hypersensitivity to $\mathrm{H}_{2} \mathrm{O}_{2}$ (Oide et al. 2006). As the homolog of NPS6 in Aspergillus nidulans and Alternaria alternata, VdNPS was first mentioned by Wang et al. (2018), who reported that nonribosomal peptide synthetases (VDAG_05314) was highly upregulated under iron sufficiency compared with that under conditions of iron starvation in $V$. dahliae XS11 strain and $\triangle V d H a p X$ strains. However, no further functional characterization of $V d N P S$ was reported later.

Verticillium dahliae Kleb. is a soilborne, hemibiotrophic, phytopathogenic fungus that causes wilt disease in over 200 species in economically important crops (Pegg and Brady 2002), such as cotton, potato, tomato, and sunflower. Every year, the global economic losses caused by Verticillium wilt exceeds $\$ 1.8$ billion. Due to the long viability of rest structures (microsclerotia), broad host range, and the limited activity of fungicide against the fungus once it enters the (root) vascular system, the disease is difficult to control (Fradin and Thomma 2006; Luo et al. 2014). During its initial biotrophic stage, the synthesis of cell-wall-degrading enzymes (CWDEs), the release of effectors, and the ability to suppress plant basal resistance contribute to the successful infection and tissue colonization of $V$. dahliae in hosts. To overcome pathogen attack, plants establish a variety of defense responses, including callose deposition (Gómez et al. 1999), oxidative burst (Apel and Hirt 2004), synthesis of pathogenesis-related (PR) proteins induced by salicylic acid (SA), jasmonic acid (JA), or ethylene (ET) (van Loon et al. 2006), and localized cell death via the hypersensitive response (Hwang and Hwang 2011; Kim and Hwang 2011).

Upon recognition of pathogen's infection, as a core component of the early plant immune response (Daudi et al. 2012), ROS burst in the apoplast (Torres 2010; Torres et al. 2006) and then diffuse into the cytoplasm and serve as signals, interacting with other signaling processes such as phosphorylation cascades, calcium signaling, and hormone-mediated pathways (Kovtun et al. 2000; Mou et al. 2003). ROS, primarily superoxide, and $\mathrm{H}_{2} \mathrm{O}_{2}$, produced by plasma membrane-localized NADPH oxidases and class III peroxidases (Doke et al. 1996; Guo et al. 2011), are regarded as one of the fastest 
defense reactions against pathogen attack (Apostol et al. 1989). However, excessively high levels of ROS can disturb the immune responses of plants and ultimately result in cellular injury and tissue damage (Krishnamurthy and Rathinasabapathi 2013; Vellosillo et al. 2010), thus weakening the resistance of plants to pathogen infection (Puertollano et al. 2011; Lorrain et al. 2003). In plant defense, ROS always occur in conjunction with other plant signaling molecules, especially SA (Torres et al. 2006). The accumulation of ROS drives the production of SA, which subsequently enhances ROS production (Shirasu et al. 1997). These two immune molecules drive the hypersensitive reaction (HR) and mediate the establishment of systemic acquired resistance (SAR) (Durrant and Dong 2004). In most plants, free SA represents a key compound in the activation of plant defense against biotrophic and hemibiotrophic pathogens (Grant and Lamb 2006), and its accumulation is necessary for the induction of $\mathrm{HR}$ and the expression of marker $P R$ genes (Alvarez 2000; Dong 2004). JA is another key hormone involved in plant immune responses to necrotrophic pathogens and herbivorous insects (Bari and Jones 2009; Ton et al. 2002). Upon hypersensitive necrosis against $V$. dahliae infection in cotton, not only does the level of SA increase, but also JA synthesis is strongly enhanced during the early stage (Grant and Jones 2009; Seo et al. 2001). Thus, during biotrophic stage, suppressing the immune system mainly the SA-dependent defenses is a strategy of $V$. dahliae to promote disease (Gkizi et al. 2016). ROS/hormone-mediated signaling pathways, resistant proteins (Gao et al. 2013b; Gao et al. 2011; Li et al. 2014, 2016; Qin et al. 2004; Sun et al. 2014; Yang et al. 2015), and intrinsic metabolites (Gao et al. 2013a; Luo et al. 2001; Tan et al. 2000; Xu et al. 2011) improve our understanding of the complicated innate defense systems of host plants against $V$. dahliae. However, little is known about the mechanism of NRPS in virulence and the relationship with host immunity. The research was aimed to illustrate the functional mechanism of VdNPS in V. dahliae by (i) demonstrating the influence of VdNPS deletion on vegetative growth and virulence of $V$. dahliae; (ii) clarifying the role of VdNPS in response to $\mathrm{H}_{2} \mathrm{O}_{2}$ stress; and (iii) exploring the function of VdNPS involved in SA tolerance and repressing host immunity.

\section{MATERIALS AND METHODS}

Strains, culture conditions, and plant lines. The defoliating type V. dahliae strain V991 was first isolated from cotton that originated in Xinjiang, China and used as a wild-type (WT) strain for transformation in this study. The strain and its transformants were stored at $-80^{\circ} \mathrm{C}$ in the form of a conidial suspension in $30 \%$ glycerol and were reactivated on potato-dextrose agar (PDA) medium at $25^{\circ} \mathrm{C}$. Conidia for infection assays were cultured in potato dextrose broth (PDB) medium. Basal media modified (BMM) was used for inducing microsclerotial development (Luo et al. 2019). Agrobacterium tumefaciens AGL-1 was used for genetic transformation, which was grown in YEB $(0.5 \%$ sucrose, $0.1 \%$ yeast extract, $1 \%$ tryptone, $\left.0.05 \% \mathrm{MgSO}_{4} \cdot 7 \mathrm{H}_{2} \mathrm{O}, \mathrm{pH} 7.0\right)$ agar/liquid medium. Cotton (Gossypium hirsutum L.) and tobacco (Nicotiana benthamiana), which were used for pathogenicity assays, were grown in growth chambers at $25^{\circ} \mathrm{C}$ with 16 -h photoperiods and $55 \%$ humidity.

Identification of positive randomly insertional transformants. The construction and identification of T-DNA randomly insertional mutants was performed as Luo et al. (2016). The randomly insertional mutants were constructed using pPK2 vector by Agrobacterium tumefaciens mediated transformation (ATMT). To detect the T-DNA insertion sites, hiTAIL-PCR (Liu and Chen 2007) was used for amplifying the flanking sequences of the positive transformants. The insertional sites were finally identified through sequencing and blasting with the genome of V. dahliae VdLs.17 strain (http:// fungi.ensembl.org/Verticillium_dahliae/Info/Index) (Klosterman et al. 2011). The primers of hiTAIL-PCR were listed in Supplementary Table S1.
Vector construction and genetic transformation. To generate the knockout plasmids pPK2-VdNPS, 892 bp of upstream and $925 \mathrm{bp}$ of downstream fragments were amplified from the genomic DNA with the primer pairs of F-U-VdNPS/R-U-VdNPS and F-D-VdNPS/R-D-VdNPS, respectively. A 3,032-bp hygromycin resistant cassette was amplified from the vector $p$ Silent-1 using primers F-P-VdNPS/R-T-VdNPS. These three fragments were fused to U-hph-D using primers F-U-VdNPS/R-D-VdNPS as described previously. The U-hph-D fragment was inserted into pPK2 binary vector by double enzyme digestion with EcoRV/XbaI. The complementation fragment, which contained the open reading fragment (ORF) of the $V d N P S$ and its native promoter region, was amplified by PCR with primers F/R-in-VdNPS, and linked into pPNK2 complementation vector by HindIII/SpeI. pPNK2 was modified from $\mathrm{pPK} 2$ by replacing the $\mathrm{Hyg} B$-resistance cassette with the Neo-resistance cassette. The knockout and complementation plasmids were transformed into Agrobacterium tumefaciens AGL1 , and the classical ATMT was performed as specified in Luo et al. (2016). Deletion mutants ( $\triangle V d N P S)$ were screened on PDA supplemented with hygromycin at $50 \mathrm{mg} / \mathrm{liter}$, and complementation transformants $(\triangle V d N P S: V d N P)$ were screened with G418 at $100 \mathrm{mg} /$ liter. All the deletion transformants were identified by PCR screening using F- $h p h / \mathrm{R}-h p h$ to confirm whether the $h p h$ resistant cassette was inserted into the genome, using F-VdNPS/R-VdNPS to confirm whether the ORF of VdNPS was deleted successfully, and using F/R-in-VdNPS to confirm whether the $H y g B$ resistant cassette was randomly inserted into the genome or replaced the target ORF of $V d N P S$ through homologous recombination. The fragment amplified using F/R-in-VdNPS was sequenced further to ensure the target gene was deleted successfully. The complementation mutants were also identified as above to check if the deletion mutant was complemented or not.

The deletion and complementation mutants of $V d S A H$ were generated as above. The fragment size of each PCR product was list in Supplementary Table S1. Furthermore, the overexpression vector of $V d S A H$ was constructed using fusion PCR. Enhanced PtrpC promotor and TtrpC terminator of Aspergillus nidulans gpdA gene were amplified from $p$ Silent-1 vector by primer pairs of F/R-P2$V d S A H$ and F/R-T2-VdSAH, respectively. F/R-GFP- $V d S A H$ was used to amplify GFP from plasmid pPHGK, and F/R-VdSAH was used to amplify the ORF of $V d S A H$ from genomic DNA, and the two fragments were fused as VdSAH-GFP using F-VdSAH/R-GFP$V d S A H$. Following, PtrpC promotor, VdSAH-GFP, and TtrpC terminator were fused to PtrpC-VdSAH-GFP-TtrpC using F-P2$V d S A H / \mathrm{R}-\mathrm{T} 2-V d S A H$. The overexpression fragment was linked into pPK2 by XbaI/HindIII digestion. All primers are listed in Supplementary Table S1.

Measuring the response of $V$. dahliae to abiotic stresses. In order to test the sensitivity of the $V$. dahliae to osmotic stress, oxygen radicals, and plant hormones, WT and transformants were cultured in PDB for 5 days and filtered through two layers of lens paper to prepare conidia suspension at $10^{7} \mathrm{spores} / \mathrm{ml}$. Three microliters of conidia suspension of WT and transformants were individually pipetted onto PDA containing $1 \mathrm{M} \mathrm{NaCl}, 1.0 \mathrm{M}$ sorbitol, $2 \mathrm{mM} \mathrm{H}_{2} \mathrm{O}_{2}, 3.5 \mathrm{mM} \mathrm{JA}$, and $3.5 \mathrm{mM} \mathrm{SA}$. Transformants and WT strains were cultured at $\mathrm{pH}=4.0$ and 12.0 to measure the effects of $\mathrm{pH}$ on their growth. Strains were also exposed to ultraviolet rays for $1 \mathrm{~h}$ to test their viability. Colony diameters were measured 7 days postinoculation (dpi). All the experiments were repeated three times. To determine whether both iron and $\mathrm{Cu}^{2+}$ were involved in the response to $\mathrm{H}_{2} \mathrm{O}_{2}, 0,1,5,10,20,30$, and $50 \mu \mathrm{M} \mathrm{Fe}^{2+}$ and $\mathrm{Cu}^{2+}$ were added to PDA medium, which contained 0 and $5 \mathrm{mM}$ $\mathrm{H}_{2} \mathrm{O}_{2}$. The growth of each colony was observed at $25^{\circ} \mathrm{C}$. Each experiment was repeated three times.

Pathogenicity assays. WT $V$. dahliae and transformants were cultured in PDB for 5 days to prepare inoculum, which was adjusted to approximately $1 \times 10^{7}$ conidia/ml. Virulence of WT, deletion mutants, overexpression mutants, and complementation mutants 
were performed on 6-week-old intact $G$. hirsutum L. and $N$. benthamiana using unimpaired root-dip inoculation method as previously described (Fradin et al. 2009; Luo et al. 2016). The fungal biomass in the infected cotton roots were quantified by semiquantitive RT-PCR as described by Luo et al. (2016). Further, in order to test the colonization of WT and transformants in soil, the tested soil was reused to measure the germination of G. hirsutum L. seeds. The plants were incubated at $25^{\circ} \mathrm{C}$ with $16-\mathrm{h} / 8$-h light/dark cycle and $55 \%$ humidity to enable disease symptoms to develop. The disease severity was evaluated by the percentage of plants that showed wilting symptom at 20 dpi after inoculation. The disease grade was classified as follows: 0 (no symptoms), 1 ( $>0$ to $25 \%$ wilted leaves), 2 ( 25 to $50 \%$ wilted leaves), 3 (50 to $75 \%$ wilted leaves), and 4 ( 75 to $100 \%$ wilted leaves). The disease index was counted as $\sum=($ number of necrosis leaves $\times$ disease grade)/(total number of leaves $\times 4) \times 100$. The virulence assays were repeated three times, and each time 12 plants were analyzed.

RNA extraction, RNA-seq, and quantitative real-time PCR (qRT-PCR). Total RNA was extracted using RNAiso Plus reagent (TaKaRa, Dalian, China) according to the manufacturer's instructions, which was free of genomic DNA. For qRT-PCR, cDNA was reverse transcribed using PrimeScript RTase (Takara, Dalian, China), and qRT-PCR was performed using iQ SYBR Green Supermix (Bio-Rad, Hercules, CA, U.S.A.) in a CFX96 thermocycler (Bio-Rad). The expression levels of target genes of $V$. dahliae were quantified relative to the constitutively expressed $\beta$-tubulin (Vdtubulin), and the transcription level of $P R$ genes (NPRl, PRl, $P R 5, P D F 1.2)$ of $G$. hirsutum was normalized using constitutively expressed histone 3 (Vdhist3). All the experiments had three independent biological replicates. Gene-specific primers designed for qRT-PCR are listed in Supplementary Table S2. To study the resistance mechanism of $V d N P S$ against ROS in $V$. dahliae, we compared the gene expression profiles between the WT and $\triangle V d N P S$ under the treatment of 0,2 , and $6 \mathrm{mM} \mathrm{H}_{2} \mathrm{O}_{2}$ for $12 \mathrm{~h}$. RNA sequencing and analysis were conducted as specified in Luo et al. (2019). The raw data were mapped to the V. dahliae VdLs.17 genome database (http://fungi.ensembl.org/Verticillium_dahliae/ Info/Index) (Klosterman et al. 2011). The RNA-seq analysis of the defense response of $G$. hirsutum against $V$. dahliae was performed similarly. After inoculation for 0, 3, 5, 7, and 9 days, the G. hirsutum roots and leaves were collected and prepared for RNA-seq. The cotton genome database (https://www.cottongen.org/organism/ Gossypium/hirsutum) (Yu et al. 2014) was used for data mapping. Data analysis and annotation were performed according to Luo et al. (2019).

ROS measurement. For detecting ROS accumulation, the roots of cotton were stained with diaminobenzidine (DAB) at $1 \mathrm{mg} / \mathrm{ml}$ as previously described (Yoshioka et al. 2003). The content of hydrogen peroxide in plant roots and leaves was measured by titanium sulfate method (Eisenberg 1943; Satterfield and Bonnell 1955). The combination of $\mathrm{H}_{2} \mathrm{O}_{2}$ and titanium sulfate (or titanium chloride) forms peroxide-titanium complex, which is yellow precipitation. The precipitation can be dissolved by $\mathrm{H}_{2} \mathrm{SO}_{4}$ and determined by colorimetry at $415 \mathrm{~nm}$ wavelength. Within a certain range, the color is linearly related to the concentration of $\mathrm{H}_{2} \mathrm{O}_{2}$.

Hoechst staining. Hoechst staining is used to visualize the nucleus integrity. Before staining the nuclei of WT and $\triangle V d N P S$, conidia were harvested from 5-day-old cultures and filtered through two layers of lens paper. Harvested conidia were treated with 0,2 , and $6 \mathrm{mM} \mathrm{H}_{2} \mathrm{O}_{2}$ for $0,12,24,48$, and $96 \mathrm{~h}$, respectively, and was recollected by centrifuging at $1,000 \mathrm{rpm}, 5 \mathrm{~min}$. The conidia were washed twice using $1 \times$ PBS buffer and were resuspended to $1 \times 10^{7}$ spores $/ \mathrm{ml}$ in $10 \mu \mathrm{g} / \mathrm{ml}$ Hoechst 33342 (Invitrogen, Shanghai, China). The suspension was incubated for $30 \mathrm{~min}$ in the dark at $25^{\circ} \mathrm{C}$ according to the kit instructions. For fluorescence images, cells were analyzed with UV/488 $\mathrm{nm}$ dual excitation, and emission was measured with standard DAPI filters on Leica confocal fluorescence microscope (Leica TCS SP8, German).
LC-MS/MS analysis. The fermentation broths of WT and $\triangle V d N P S$ were collected after $3,5,7$, and 9 days and were filtered to eliminate fungi. The total metabolites were extracted with ethyl acetate and dissolved in methanol. Internal standard substances (2-chlorphenylalanine) were added followed by vortexing for $30 \mathrm{~s}$, and then underwent ultrasonic disruption for $10 \mathrm{~min}$ on ice and incubation for $1 \mathrm{~h} \mathrm{at}-20^{\circ} \mathrm{C}$ to precipitate metabolites. Centrifugation was performed at $13,000 \mathrm{rpm}$ for $15 \mathrm{~min}$ at $4^{\circ} \mathrm{C}$, and the supernatant was prepared for ultra high performance liquid chromatography-quadruple time of flight-mass spectrometry (UHPLC-QTOF-MS) analysis. Metabolome data were processed using R package XCMS (version 3.2) (Fraga et al. 2010).

Bioinformatic and statistical analysis. Conserved domains of proteins were analyzed on online Pfam (http://pfam.xfam.org/) (El-Gebali et al. 2018). Phylogenic tree was constructed using MEGA6.0 by the neighbor-joining method. The bootstrap replicate is 1,000 . The heatmap was drawn by HemI software (Heatmap Illustrator, version 1.0) (Deng et al. 2014). Statistics were performed using IBM SPSS software. The differences among independent groups were determined by one-way analysis of variance and Student's $t$ test. The significance level was chosen to be $* P<0.05$ and $* * P<0.01$.

\section{RESULTS}

Identification and characterization of VdNPS in $\mathbf{V}$. dahliae. VdNPS (EGY14150) is annotated as N-(5-amino-5-carboxypentanoyl)L-cysteinyl-D-valine synthase in $V$. dahliae VdLs.17 genome database. Functional domain analysis of the protein indicated that VdNPS contains three kinds of conserved domains (Fig. 1A): one is a typical AMP binding domain for adenylation (Pfam00501; A); the two other domains are a PP binding domain for phosphopantetheine attachment (Pfam00550; PP), and a condensation domain catalyzing amide bond formation (Pfam00668; C), which appear in conjunction as PP-C. The A domain occurs in triplicate, and the PP-C domain repeats six times in VdNPS. Based on the published architecture of NRPSs (Marahiel 2016; Tarry and Schmeing 2015), the conserved domains were organized to form three modules to incorporate each amino acid into the end product $(\delta$ - $(\mathrm{L}-\alpha$-aminoadipyl)-L-cysteine-Dvaline, $\mathrm{C}_{14} \mathrm{H}_{25} \mathrm{~N}_{3} \mathrm{O}_{6} \mathrm{~S}$, molecular mass: 363.65 ) (Fig. 1A). Phylogenetic analysis indicated that VdNPS is an ortholog of NPS6 in F. graminearum (identity: $76.4 \%$ ) and Alternaria brassicicola (identity: $70.4 \%$ ) (Fig. 1B). VdNPS was firstly isolated through T-DNA random insertion. The inserted mutant (IM $\triangle V d N P S)$ showed decreased virulence and microsclerotial formation (Supplementary Fig. S1A and B). Through sequencing the right-border flanking sequence, the mutant was identified to disrupt the encoding region of the fourth condensation domain of VdNPS (Supplementary Fig. $\mathrm{S} 1 \mathrm{C})$. Because the left-border flanking sequence was amplified unsuccessfully, we constructed knockout and complementation mutants through homologous recombination for further research.

Deletion and complementation mutants of VdNPS. Owing to the large ORF of $V d N P S(15,337 \mathrm{bp})$, it was difficult to replace the entire ORF with $H y g B$ resistance cassette. Thus, we replaced only the $2,500 \mathrm{bp}$ of the ORF from the initiation codon, which contained an integral set of domain (A-PP-C). Subsequent molecular verification of transformants showed that three colonies (No. 2, No. 3, and No. 10) were deleted successfully (Supplementary Fig. S2A and C), which were named $\triangle V d N P S-2, \triangle V d N P S-3$, and $\triangle V d N P S$-10, respectively. The deletion mutant $\triangle V d N P S$-2 was further used for constructing complementation mutant, and $\triangle V d N P S-2$ and $\triangle V d N P S-3$ were used for further measurement of pathogenicity and abiotic stress response. Through PCR amplification and sequencing, the No. 2 transformant was confirmed as the complementation mutant, annotated as $\triangle V d N P S: V d N P S$ (Com) (Supplementary Fig. S2B and C).

Decreased conidiation and microsclerotial formation in $\triangle V d N P S$ and its response to abiotic stress. Although there was no difference among $\triangle V d N P S$, Com, and WT with respect to 
colony diameter and growth rate, the formation of melanized microsclerotia in $\triangle V d N P S$ was much weaker than that in Com and WT (Fig. 2A and B). In addition, the $\triangle V d N P S$ mutants produced significantly fewer conidia than WT and Com $(P<0.05)$ (Fig. 2C).
Further abiotic stress response showed that under $1.0 \mathrm{M} \mathrm{NaCl}, 1.0 \mathrm{M}$ sorbitol, ultraviolet radiation, $\mathrm{pH} 4.0$, and $\mathrm{pH} 12.0$ conditions, the vegetative growth of the $\triangle V d N P S$ mutant was not significantly different to that of Com and WT strains. However, the $\triangle V d N P S$
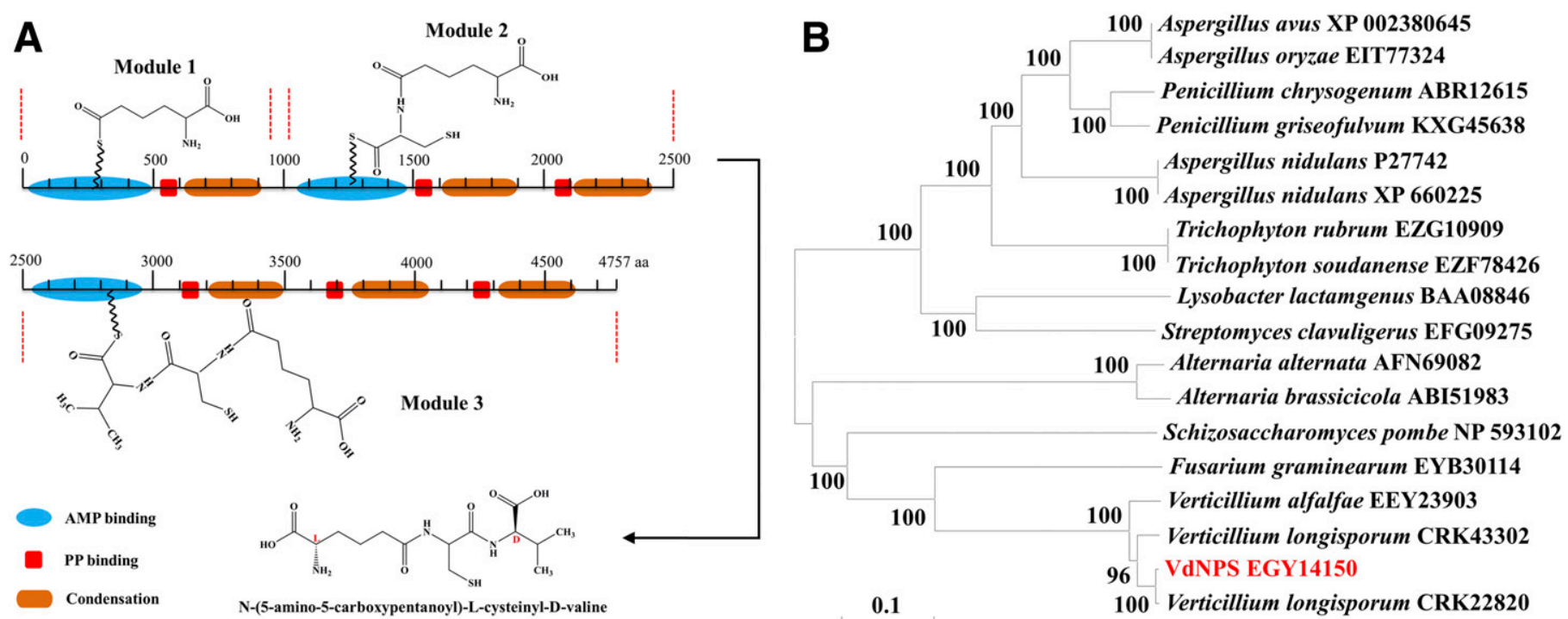

Fig. 1. The characterization of nonribosomal peptide synthetases (NPS) in Verticillium dahliae. A, Overall architecture of VdNPS shows three different modules, which incorporate amino acids into the end product ( $\delta$-(L- $\alpha$-aminoadipyl)-L-cysteine-D-valine). Module boundaries are defined based on Pfam analysis and previous reports (Marahiel 2016; Tarry and Schmeing 2015). Each extension module comprises three core domains: one for adenylation (A, shown in blue), one for catalyzing peptide bond formation (C, shown in orange), and one for phosphopantetheine attachment (PP, shown in red). B, Phylogenetic relationship among nonribosomal peptides (NRPs) from different fungi. The amino acid sequences of 18 NRPs from public databases (https://www.ncbi.nlm.nih.gov) distributed into two clades showed high level of similarity. The percentage of trees in which the associated proteins clustered together (at least $50 \%$ in 1,000 bootstrap replicates) is shown next to the branches. The phylogenetic tree was constructed using MEGA6.0 by the neighbor-joining method.

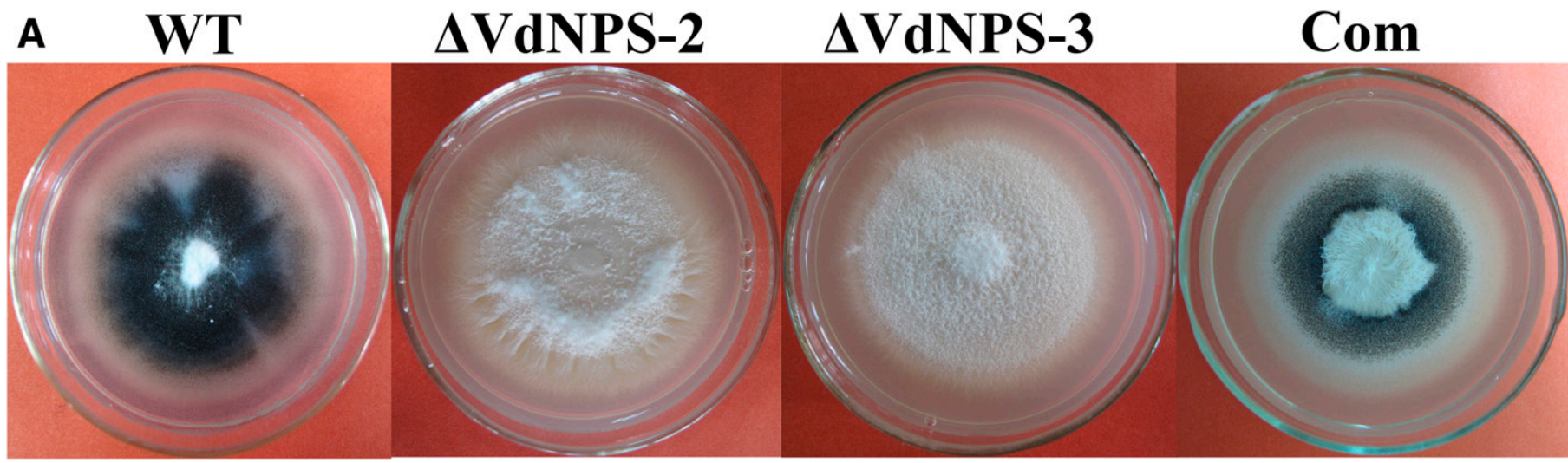

B

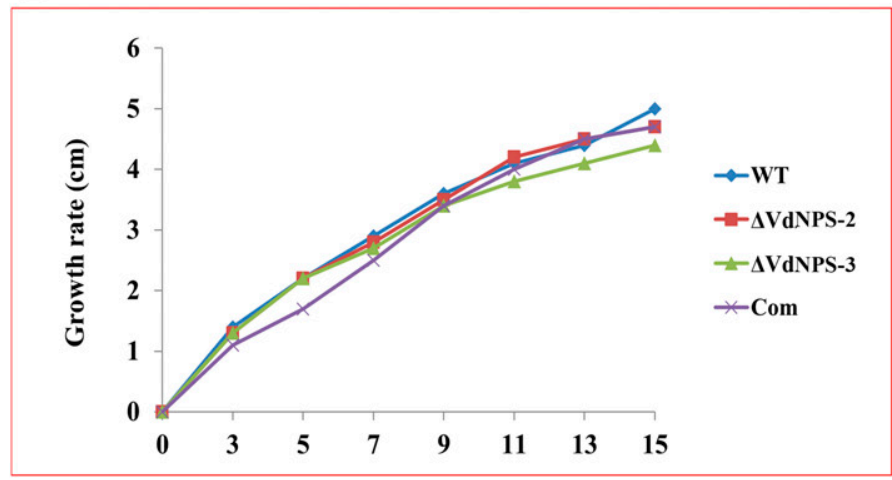

C

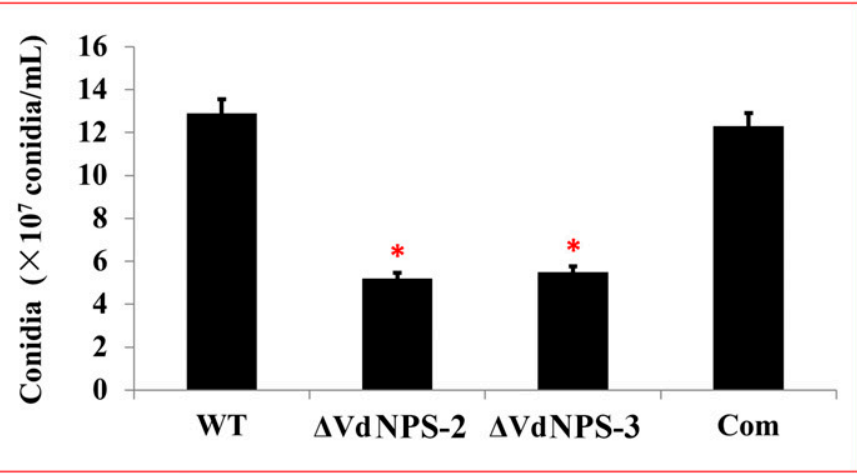

Fig. 2. Phenotype of $\triangle V d N P S$. A, The colony phenotype of $\triangle V d N P S$. Reduced microsclerotia formed in $\triangle V d N P S$. B, No significant difference existed in the growth rate of $\triangle V d N P S$ mutants and wild type (WT). The $x$-axis represents growth days. $\mathbf{C}$, The decreased conidiation in $\triangle V d N P S$. The statistical analysis was performed using $t$ test $(* P<0.05)$. The experiment was repeated three times $(n=3)$. 
showed significant resistance to $\mathrm{H}_{2} \mathrm{O}_{2}$ and hypersensitivity to SA and JA (Supplementary Fig. S3).

The analysis of metabolites in $\triangle V d N P S$ mutants. After fermenting for 7 days, total metabolites of WT and $\triangle V d N P S$ were analyzed by HPLC-MS. The results showed the mass-spectrum peak of 363.7 ( $\delta$-(L- $\alpha$-aminoadipyl)-L-cysteine-D-valine) did not exist in the $\triangle V d N P S$ mutant (Fig. 3A). Further metabolic profiling indicated that compared with WT, 213, 179, 142, and 103 metabolites were differentially regulated in $\triangle V d N P S$ at 3, 5, 7, and 9 days, respectively (Fig. 3B). The differentially regulated metabolites were enriched in the biosynthesis of secondary metabolites (ko01110), and biosynthesis of antibiotics (ko01130) (Fig. 3C to F); and over $66.7 \%$ differentially regulated metabolites in these pathways were down-regulated in $\triangle V d N P S$ (Supplementary Table S3). It is worth noting that no metabolite associated with siderophores was detected in both WT and $\triangle V d N P S$, while the biosynthesis of $\beta$-lactam antibiotics carbapenem (ko00332) and staurosporine (ko00404) were enriched in $\triangle V d N P S$ (Fig. 3C to F). Carbapenems are used to treat serious infections caused by extended-spectrum $\beta$-lactamase carrying pathogens (Gupta et al. 2011). L-Proline and L-glutamate were differentially synthesized metabolites involved in the carbapenem biosynthesis, which were down-regulated in $\triangle V d N P S\left(P<0.05, \log _{2} \mathrm{FC}<0\right)$ (Supplementary Table S3). The antibiotics staurosporine is an inhibitor of phospholipid/Ca ${ }^{++}$-dependent protein kinase, and it could induce programmed cell death (Jacobsen et al. 1996; Tamaoki et al. 1986). L-Proline is a key protein in staurosporine biosynthesis, which was down-regulated in $\triangle V d N P S$ at 3, 5, and 7 days (Supplementary Table S3). The results indicated that VdNPS is more likely an enzyme for metabolite biosynthesis than for siderophore production as NPS6 in Alternaria alternata, C. heterostrophus, $F$. graminearum, and Alternaria brassicicola (Chen et al. 2013; Oide et al. 2006). However, the biosynthesis of specific metabolites and antibiotics mediated by VdNPS need further isolation and identification.

VdNPS deletion decreased $\mathrm{H}_{2} \mathrm{O}_{2}$ sensitivity. In vitro tests indicated that $\triangle V d N P S$ strains exhibited decreased sensitivity to different concentrations of $\mathrm{H}_{2} \mathrm{O}_{2}$ than WT. The IC50 and lethal concentration of $\mathrm{H}_{2} \mathrm{O}_{2}$ was 2 and $4 \mathrm{mM}$ in WT, respectively; while those in $\triangle V d N P S$ was 4 and $6 \mathrm{mM}$, individually (Fig. 4A and Supplementary Fig. S4A). To elucidate the molecular mechanism of $\mathrm{H}_{2} \mathrm{O}_{2}$ tolerance in the $\triangle V d N P S$ strain, we examined the gene expression by transcriptional profiling analysis under the stress of 0 , 2 , and $6 \mathrm{mM} \mathrm{H}_{2} \mathrm{O}_{2}$. Results showed that the genes encoding cell wall protein and cytoskeleton complex protein were significantly upregulated in $\triangle V d N P S$, while the genes encoding membrane intrinsic protein were significantly down-regulated (Supplementary Table S4). Additionally, genes encoding DNA repair protein were significantly up-regulated in $\triangle V d N P S$ (Supplementary Table S4), and Hoechst staining analysis indicated that high concentration of $\mathrm{H}_{2} \mathrm{O}_{2}$ led to nuclear degradation in $\triangle V d N P S$ and WT (Supplementary Fig. S5). NADPH oxidases (Nox) is the major enzymatic producer of ROS (Egan et al. 2007), which is important for the appressoria formation, while the VDAG_00930 encoding NoxB was down-regulated under $\mathrm{H}_{2} \mathrm{O}_{2}$ stress both in WT and $\triangle V d N P S$; and compared with WT, the expression of NoxB was downregulated in $\triangle V d N P S$. Importantly, the expression levels of three categories of genes associated with $\mathrm{H}_{2} \mathrm{O}_{2}$ detoxification were differentially expressed under $\mathrm{H}_{2} \mathrm{O}_{2}$ stress in $\triangle V d N P S$, including cat (VDAG_09115), sod (VDAG_00019, VDAG_07230, VDAG_ 08724, VDAG_09837), and pod (VDAG_03116). Without $\mathrm{H}_{2} \mathrm{O}_{2}$, compared with WT, VDAG_00019, VDAG_07230, and VDAG_ 09115 were up-regulated by 9-fold, 3.9-fold, and 3.82-fold in $\triangle V d N P S$, respectively. Under the stress of $2 \mathrm{mM} \mathrm{H}_{2} \mathrm{O}_{2}, \mathrm{VDAG}_{-}$ 00019 and VDAG_07230 were up-regulated by 2.86-fold and 3.12fold in $\triangle V d N P S$, respectively (Fig. 4B). These data suggested that

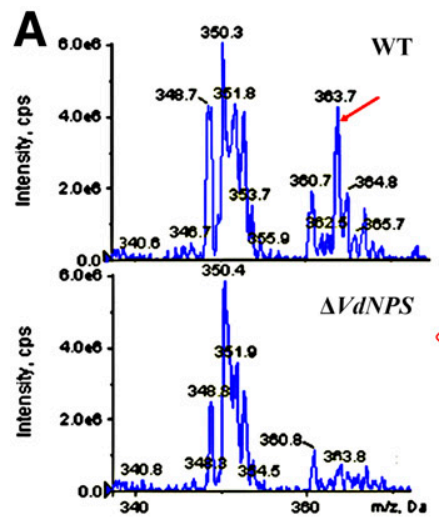

\section{B}

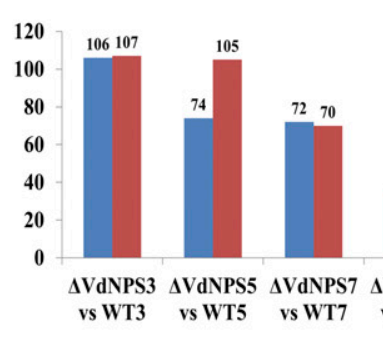

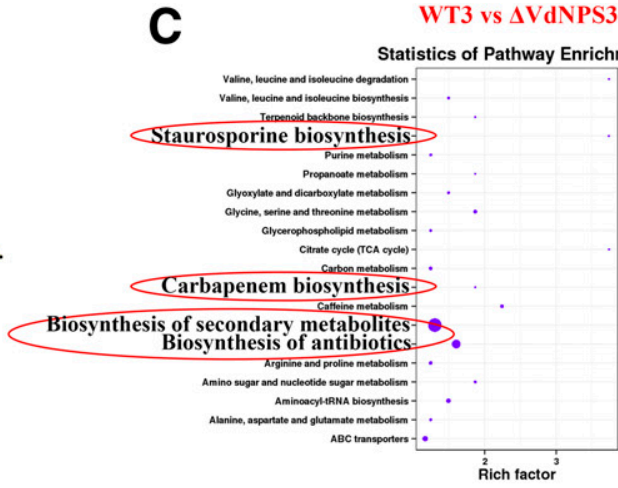

E
WT7 vs $\triangle$ VdNPS7

Statistics of Pathway Enrichmen
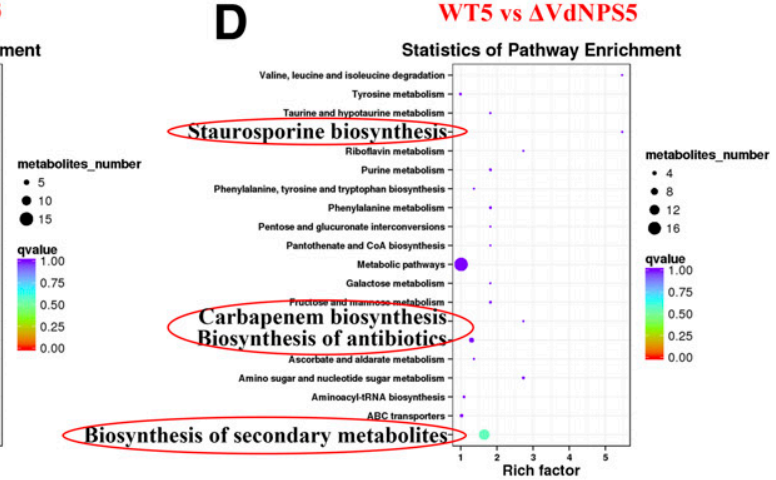

F

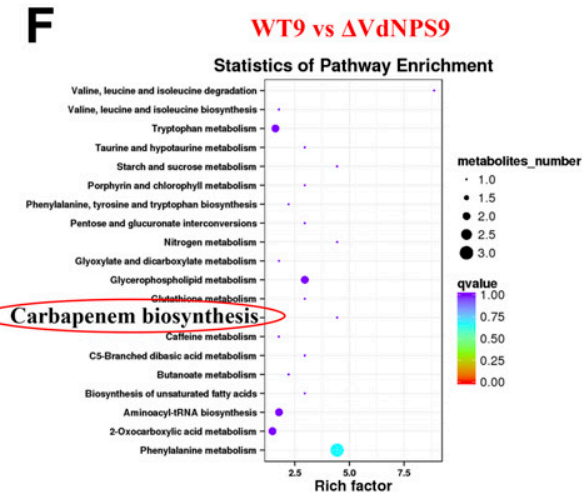

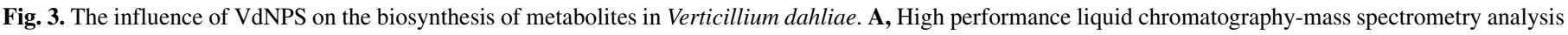

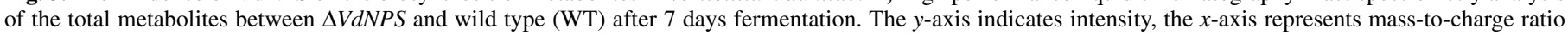

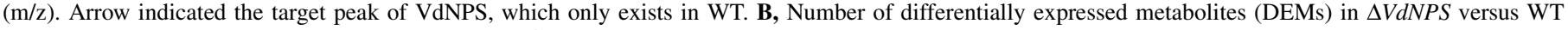

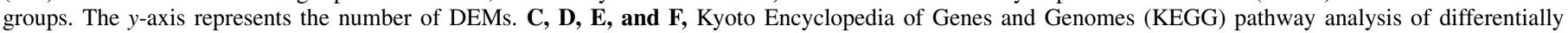

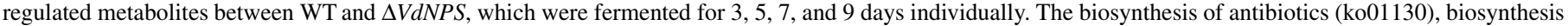
of secondary metabolites (ko01110), staurosporine biosynthesis (ko00404), and carbapenem biosynthesis (ko00332) were enriched in DEMs. 
$\triangle V d N P S$ alleviated oxidative stress by regulating the expression of genes associated with strengthening cell structure, detoxifying $\mathrm{H}_{2} \mathrm{O}_{2}$, and inhibiting ROS production in vivo.

VdNPS involved in the response to $\mathrm{H}_{2} \mathrm{O}_{2}$ stress via iron/ copper. $\mathrm{H}_{2} \mathrm{O}_{2}$, function in the form of high reactive hydroxyl radical $\left(\mathrm{OH}^{\bullet}\right)$. During the process of transforming $\mathrm{H}_{2} \mathrm{O}_{2}$ to $\mathrm{OH}^{\bullet}$, $\mathrm{Fe}^{2+}$ release and reduce to $\mathrm{Fe}^{3+}$, and stimulates $\mathrm{O}^{2-}$ transformation to $\mathrm{OH}^{\bullet}$ through the Fenton reaction (Kohanski et al. 2010, 2007). Unexpectedly, during the response to $\mathrm{H}_{2} \mathrm{O}_{2}$, transcriptome sequencing revealed that besides iron, copper was also involved in the response to $\mathrm{H}_{2} \mathrm{O}_{2}$. Under the treatment of 0,2 , and $6 \mathrm{mM} \mathrm{H}_{2} \mathrm{O}_{2}$, genes encoding siderophore iron transporter (VDAG_03807 and VDAG_08042) were down-regulated in $\triangle V d N P S$, but genes encoding ferric reductase (VDAG_03652 and VDAG_06705) were up-regulated in the presence of $2 \mathrm{mM} \mathrm{H}_{2} \mathrm{O}_{2}$, and VDAG_02046 was down-regulated in the presence of $6 \mathrm{mM} \mathrm{H}_{2} \mathrm{O}_{2}$ (Fig. 4D). As the cofactor of $\mathrm{Cu} / \mathrm{Zn} \mathrm{SOD}, \mathrm{Cu}$ could induce cellular antioxidants scavenged ROS under optimal level (Baker 1987), and excessive $\mathrm{Cu}$ could catalyze the production of ROS (Aust et al. 1985). Contrary to iron related transporter and enzymes, genes encoding copper transporter (VDAG_06704, VDAG_06151) and copper amine oxidase (VDAG_07314) were up-regulated under the stress of 2 and $6 \mathrm{mM} \mathrm{H}_{2} \mathrm{O}_{2}$ (Fig. 4D).
Due to an important role of iron/copper in detoxification of oxidative stress, we assessed whether deletion of VdNPS affected $\mathrm{H}_{2} \mathrm{O}_{2}$ sensitivity through iron/copper uptake. Results showed that under normal condition, exogenous application of lower concentration $(<30 \mu \mathrm{M})$ of $\mathrm{Fe}^{2+}$ and $\mathrm{Cu}^{2+}$ did not affect the growth of $\triangle V d N P S$ and WT. In the presence of $5 \mathrm{mM} \mathrm{H}_{2} \mathrm{O}_{2}$, exogenous application of $\mathrm{Fe}^{2+}(<10 \mu \mathrm{M})$ and $\mathrm{Cu}^{2+}(<30 \mu \mathrm{M})$ relieved the $\mathrm{H}_{2} \mathrm{O}_{2}$ damage to WT; while the iron/copper-excess did not affect the growth of $\triangle V d N P S$ under $\mathrm{H}_{2} \mathrm{O}_{2}$ stress (Fig. $4 \mathrm{C}$ and Supplementary Fig. S4B to E). The results implied that VdNPS is essential for $V$. dahliae to confront oxidative stress, and iron/copper regulate $\mathrm{H}_{2} \mathrm{O}_{2}$ response coordinately.

$\Delta V d N P S$ strains exhibited hypersensitivity to SA via upregulation of $\mathrm{SA}$ hydroxylation-associated genes. $\mathrm{SA}$ is a multitasking and pleiotropic molecule, which could induce plant immune responses and has direct antifungal activity. In vitro inhibition tests showed that $\triangle V d N P S$ strains were hypersensitive to SA (Fig. 5A and B). To further explore the function of VdNPS in response to SA stress, we monitored the expression of key genes involved in SA hydroxylation. The hydroxylation of SA leads to loss of function, which regulates the excessive accumulation of SA and maintains SA homeostasis (Zhang et al. 2017). Four genes associated with SA hydroxylation exist in $V$. dahliae, including
A

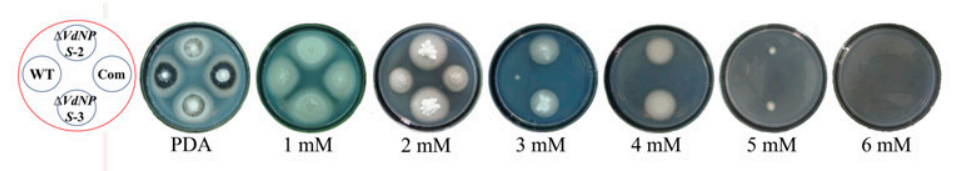

B

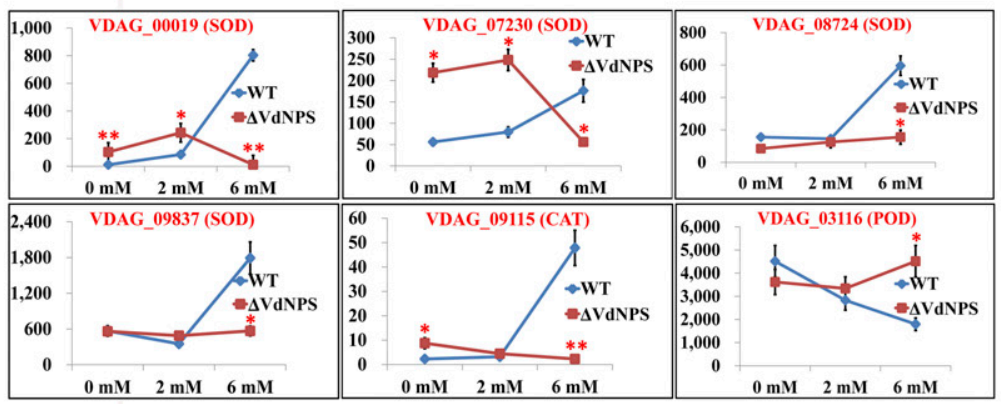

C

$\mathrm{Cu}^{2+}$
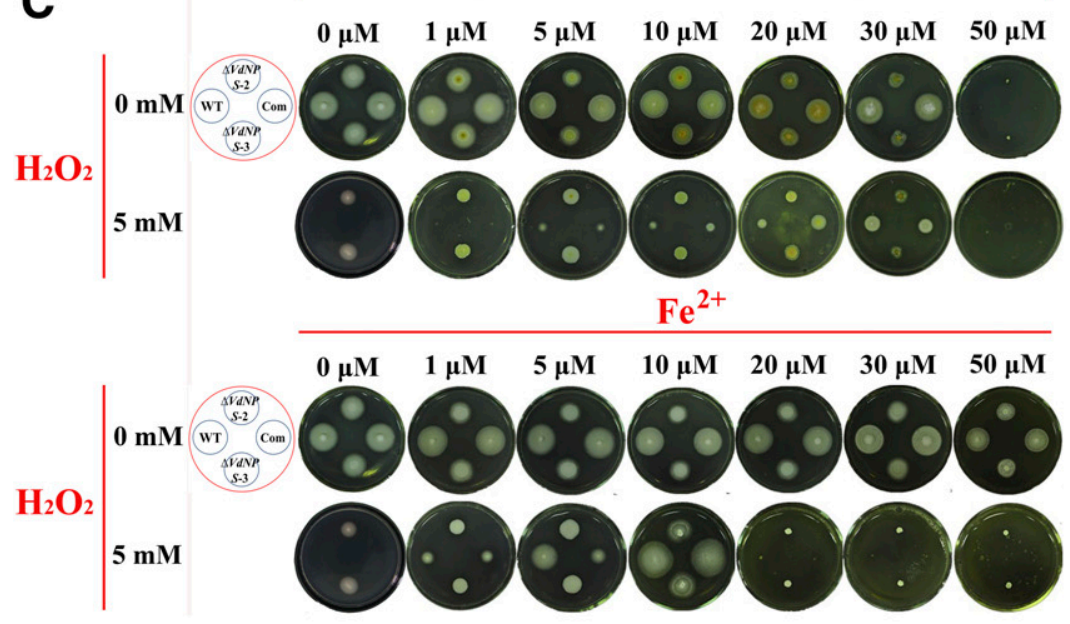

D

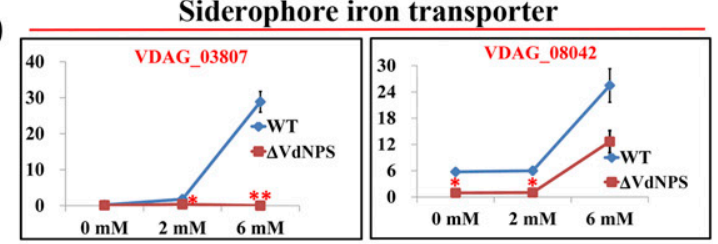

High affinity copper transporter

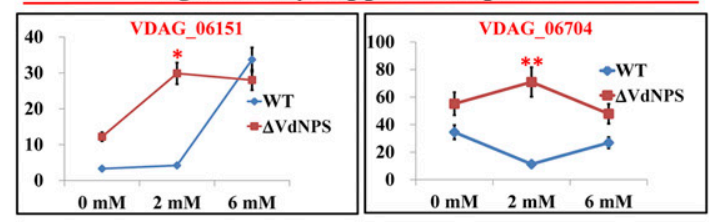

Copper amine oxidase

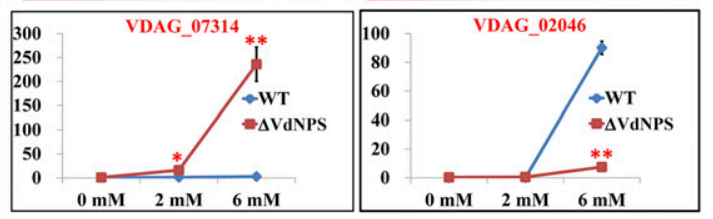

Ferric reductase

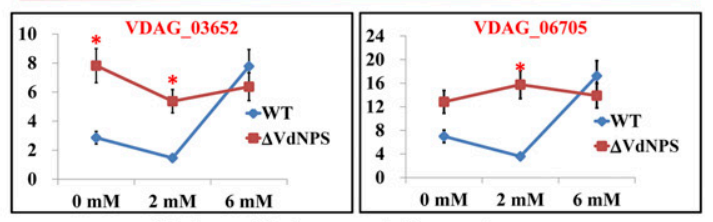

Extracellular metalloprotease

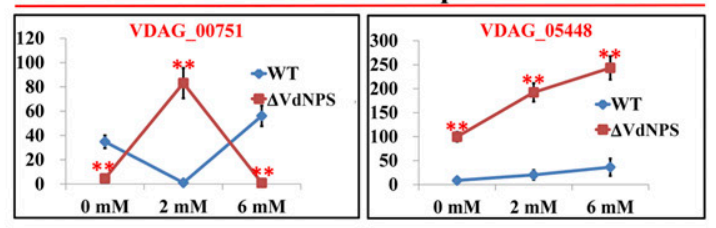

Fig. 4. The response mechanism of $\triangle V d N P S$ to $\mathrm{H}_{2} \mathrm{O}_{2}$ stress. A, Growth inhibition of $\triangle V d N P S$, wild type (WT) and complementation mutant (Com) under different concentrations of $\mathrm{H}_{2} \mathrm{O}_{2}$. The deletion of $V d N P S$ decreased the sensitivity to $\mathrm{H}_{2} \mathrm{O}_{2}$ stress in $V$. dahliae. B, Normalized expression of genes encoding catalase (CAT), superoxide dismutase (SOD), and peroxidase (POD) in WT and $\triangle V d N P S$ under the stress of 0, 2, and $6 \mathrm{mM} \mathrm{H}_{2} \mathrm{O}_{2}$. CAT: VDAG_09115; SOD: VDAG_00019, VDAG_07230, VDAG_08724, and VDAG_09837; and POD: VDAG_03116. C, Colony phenotype of tested strains under exogenous application of Fe ${ }^{2+}$ and Cu ${ }^{2+}$. Exogenous application of lower concentration $(<30 \mu \mathrm{M})$ of $\mathrm{Fe}^{2+}$ and $\mathrm{Cu}^{2+}$ did not affect the growth of $\Delta V d N P S$ and WT, while in the presence of $5 \mathrm{mM} \mathrm{H}_{2} \mathrm{O}_{2}$, exogenous application of $\mathrm{Fe}^{2+}(<10 \mu \mathrm{M})$ and $\mathrm{Cu}^{2+}(<30 \mu \mathrm{M})$ relieved the $\mathrm{H}_{2} \mathrm{O}_{2}$ damage to WT. D, Normalized expression of genes encoding copper/siderophore iron transporter, ferric reductase transmembrane component, and metalloprotease in WT and $\triangle V d N P S$ under the treatment of 0,2 , and 6 mM $\mathrm{H}_{2} \mathrm{O}_{2}$. The $y$-axis indicates the relative transcripts compared with the housekeeping gene Vdtubulin. Bars represent the standard error from three independent replicates. Asterisks indicate significant differences $(* P<0.05 ; * * P<0.01 ; t$ test) between $\triangle V d N P S$ and WT. 
salicylate hydroxylase VdSAH1 (VDAG_04960), salicylate hydroxylase VdSAH2 (VDAG_07329), salicylate hydroxylase VdSAH3 (VDAG_09318), and salicylate 1-monooxygenase VdSAH4 (VDAG_01968). The four proteins are nonsecretory. The quantitative RT-PCR analysis showed that the transcript levels of the $V d S A H 1, V d S A H 2, V d S A H 3$, and $V d S A H 4$ were significantly up-regulated in $\triangle V d N P S$ under SA stress compared with those in WT on the same conditions (Fig. 5C). Further functional characterizations of $V d S A H s$ showed that the knockout mutants ( $\triangle V d S A H 1-4)$ were hypersensitive to SA, while the overexpression mutants (PtrpC::SAH1-4) were tolerant to SA (Fig. 5D and Supplementary Fig. S6). The same phenomenon was observed under JA and $\mathrm{H}_{2} \mathrm{O}_{2}$ stress (Supplementary Figs. S6 and S7A). Additionally, the deletion of VdSAH1-4 significantly weakened the virulence of $V$. dahliae, and the disease indexes were lower than $12.5 \%$, while those of overexpression strains, Com, and WT exceeded $85 \%$ (Fig. 5E to F). During infection, $\triangle V d S A H 1-4$ strains induced higher expression of $N P R 1, P R 1, P R 5$, and $P D F 1.2$ in cotton than that induced by WT, overexpression strains, and Com (Supplementary Fig. S7B). The results indicated that $\triangle V d N P S$ was hypersensitive to SA through mediating SA hydroxylation via general down-regulation of salicylate hydroxylase and salicylate 1monooxygenase, thus, to weaken the virulence of $V$. dahliae.

Reduced virulence of $\triangle \boldsymbol{V} \boldsymbol{d N P S}$ mutants. G. hirsutum and $N$. benthamiana were used to test the pathogenicity of $V$. dahliae. Twenty days after inoculation, the plants infected by Com and WT showed severe wilting symptoms (disease index, DI > 95\%) (Fig. $6 \mathrm{~A}$ ); while the intact plants infected by $\triangle V d N P S$ grew normally and the DI $(17.5 \%)$ was significantly lower $(P<0.01)$ (Fig. 6B). Semiquantitative RT-PCR analysis showed that the biomass of WT, Com, and $\triangle V d N P S$ in the cotton shoots was not significantly different (Fig. 6C). Compared with the expression in cotton inoculated with WT, the expressions of NPR1, PR1, PR5, and $P D F 1.2$ at 9 dpi were significantly up-regulated in cotton inoculated with $\triangle V d N P S$ (Fig. 6D). Diaminobenzidine (DAB) staining was carried out on cotton roots to detect the production of $\mathrm{H}_{2} \mathrm{O}_{2}$ after inoculating for 9 days. The results showed that the roots inoculated with $\triangle V d N P S$ were stained dark brown, which was a deeper color than those inoculated with WT and Com (Fig. 6E). The $\mathrm{H}_{2} \mathrm{O}_{2}$ content reached $72.248 \mathrm{~mol} / \mathrm{g}$ (fresh weight) in the roots inoculated with $\triangle V d N P S$, which was significantly higher than that observed in response to WT $(P<0.01)$ (Fig. 6E).

A

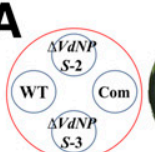
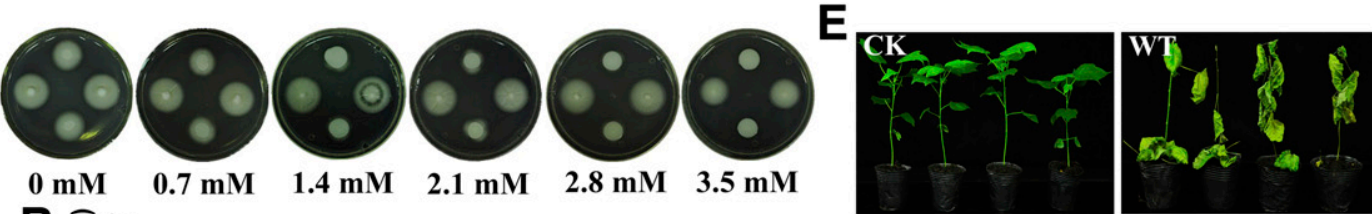

B

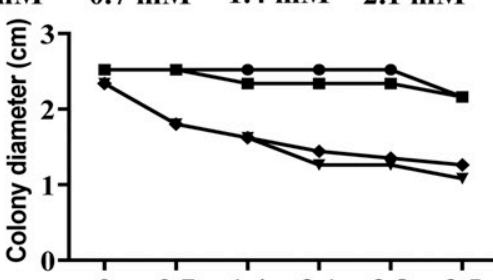

$\rightarrow W T$

$\rightarrow$ Com

$\rightarrow \triangle V D N P S-2$

$\rightarrow \triangle V d N P S-3$

$\begin{array}{llllll}0 & 0.7 & 1.4 & 2.1 & 2.8 & 3.5 \\ \end{array}$
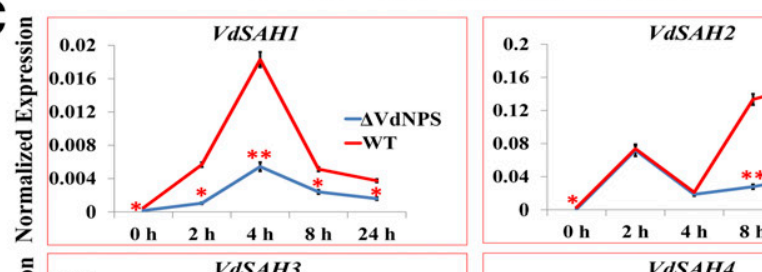

0.2
0.16

0.16
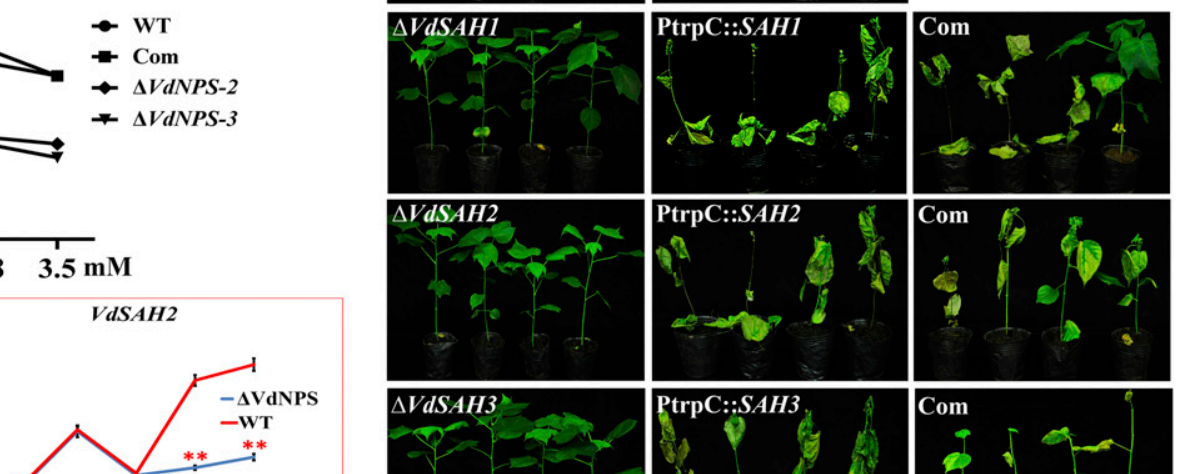

0.04

$\begin{array}{lllll}0 \text { h } & 2 h & 4 h & 8 h & 24 h\end{array}$
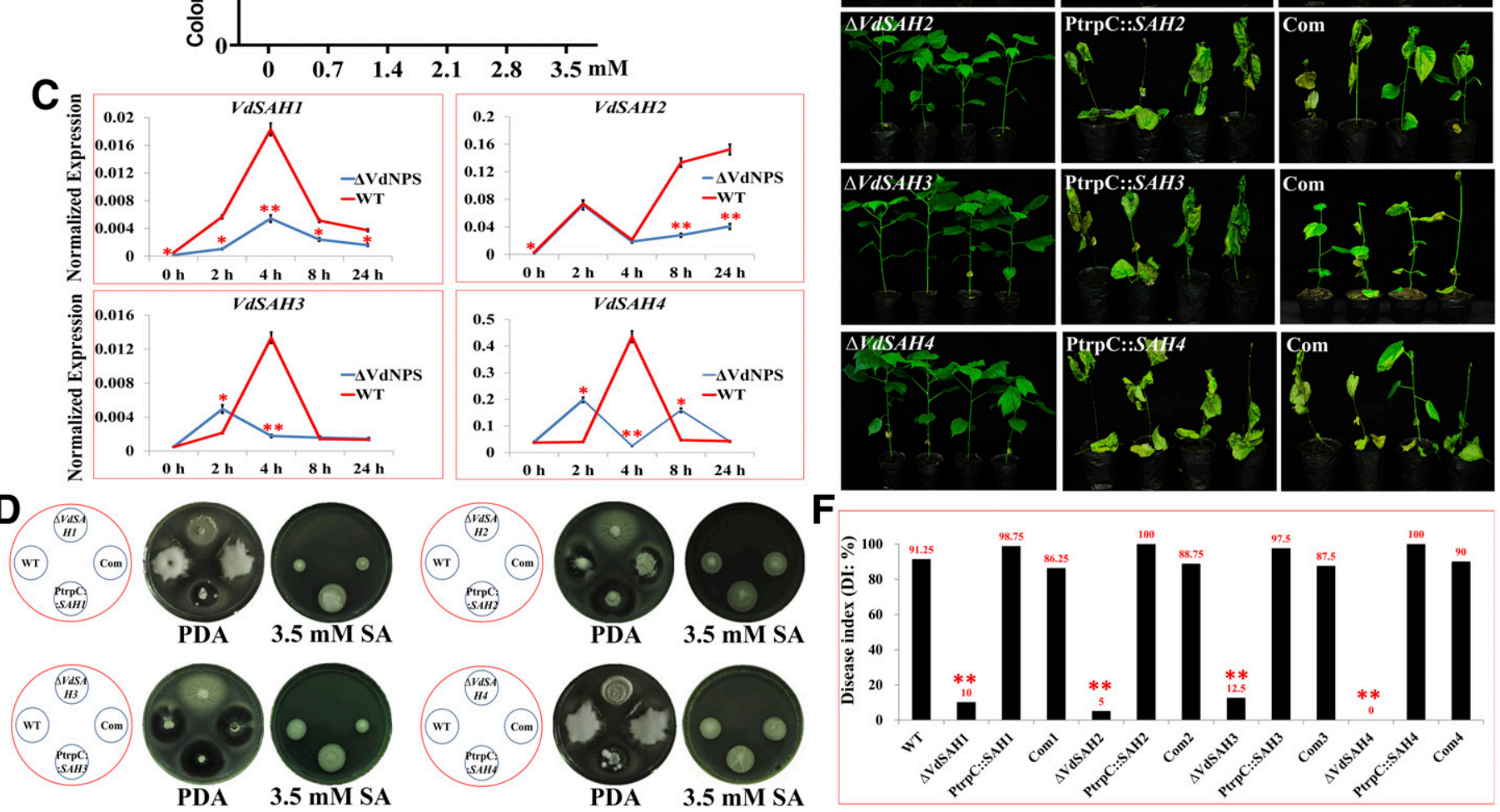

$\mathbf{F}$

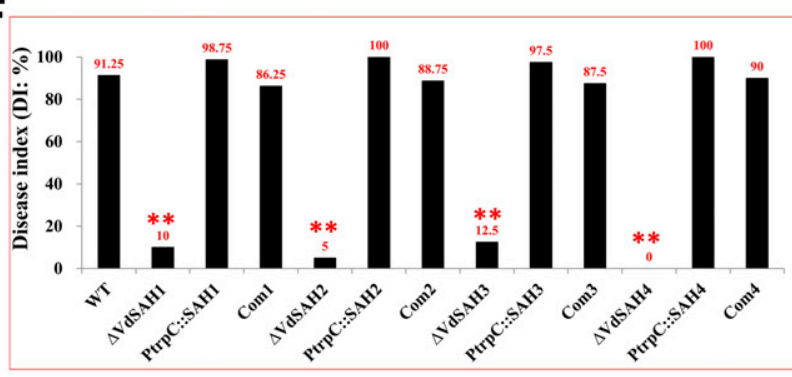

Fig. 5. $\triangle V d N P S$ strains exhibited hypersensitivity to salicylic acid (SA) via up-regulation of SA hydroxylation-associated genes. A, $\triangle V d N P S$ was hypersensitive to SA. B, The inhibited colony growth of $\triangle V d N P S$ mutants under the SA stress. C, Normalized expression of VdSAH1, VdSAH2, VdSAH3, and VdSAH4 under $3.5 \mathrm{mM}$ SA treatment for $0,2,4,8$, and $24 \mathrm{~h}$ in Verticillium dahliae. Expression was normalized against housekeeping gene Vdtubulin. D, Phenotype of VdSAH mutants under the treatment of SA. Compared with wild type (WT) and complementation mutant (Com), the knockout mutants $\triangle V d S A H$ were unable to grow in the presence of SA. E, $V d S A H$ is required for full $V$. dahliae virulence. The disease symptoms on intact cotton plants were scored at 20 days postinoculation with indicated strains. F, The disease indexes of $\triangle V d S A H$ mutants were lower than 12.5\%. $\triangle V d S A H 1-4$ represent knockout mutants; PtrpC::SAH1-4 represent overexpression mutants under the Aspergillus nidulans trpC promoter; and Com1-4 represent complementation mutants of VdSAH1-4 using its own promoter, respectively. Three biological replicates $(n=3)$ were used for this study. The statistical analysis was performed using a $t$ test $(* P<0.05 ; * * P<0.01)$. 
The host response against $\triangle V d N P S$ strain and WT V. dahliae. In order to illustrate the phenomenon of reduced virulence in $\triangle V d N P S$, we monitored the changes of transcriptional level in host infected by $\triangle V d N P S$ and WT. At $9 \mathrm{dpi}$, the cotton plants infected by WT $V$. dahliae started to wilt, while those infected by $\triangle V d N P S$ were still healthy. The transcriptional profile and Kyoto Encyclopedia of Genes and Genomes (KEGG) enrichment analysis showed that during the infection, plant hormone signal transduction (ko04075) and plant-pathogen interaction (ko04626) enriched after 5-day-inoculation (Supplementary Fig. S8, Supplementary Table S5). With respect to plant hormone signal transduction (ko04075), JA- and SA-mediated signaling pathways were significantly enriched for defense response. Compared with WT, the differentially expressed genes (DEGs) involved in JA- and SA-mediated signaling pathways were all up-regulated in $\triangle V d N P S$ at 5 dpi (Fig. 7A), including jasmonate-zim domain family proteins (JAZ) (evm.TU.Gh_ A03G1341 and evm.TU.Gh_D02G1776) and basic-helix-loop-helix transcription factors MYC2 (evm.TU.Gh_A08G1412, evm.TU.Gh_ A08G0962, and evm.TU.Gh_D08G1707) encoding genes in JAmediated signaling pathway, basic/leucine zipper-type transcription factors TGA (evm.TU.Gh_A09G0903, evm.TU.Gh_A09G1184, evm.TU.Gh_D07G1078, and evm.TU.Gh_D09G1188), and PR1 (evm.TU.Gh_A12G0274) encoding genes in SA-mediated signaling pathway (Fig. 7F). At 7 dpi, DEGs encoding JAZ were up-regulated,
A
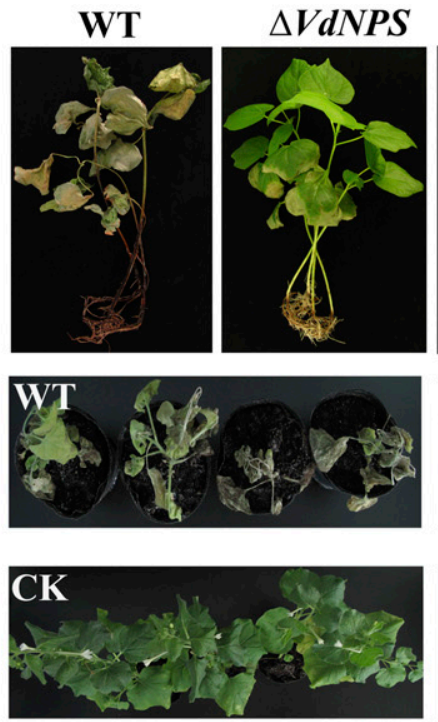

C

Vd.ITS WT

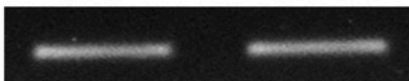

CK
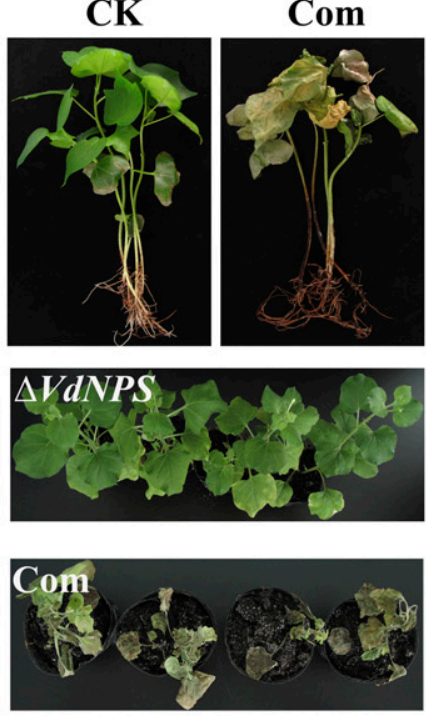

CK
Com

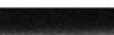

\section{Osmotin}

$-$

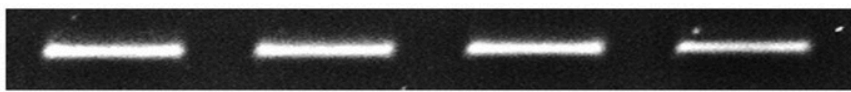

E

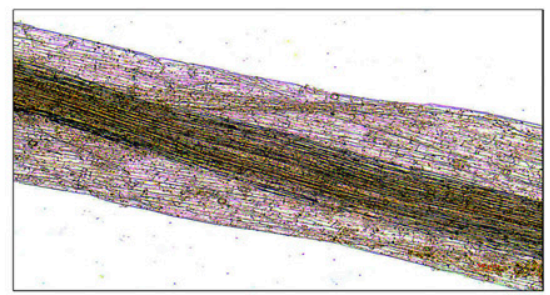

CK*

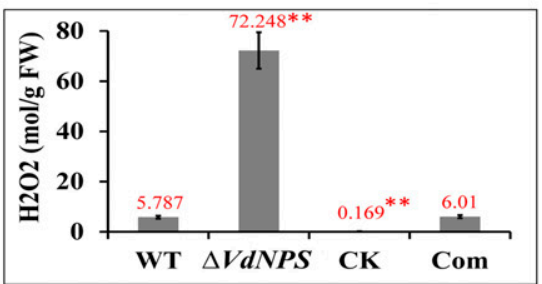

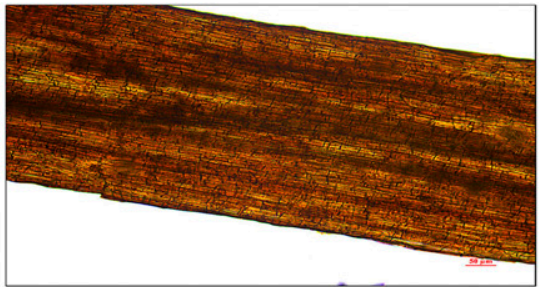

WT

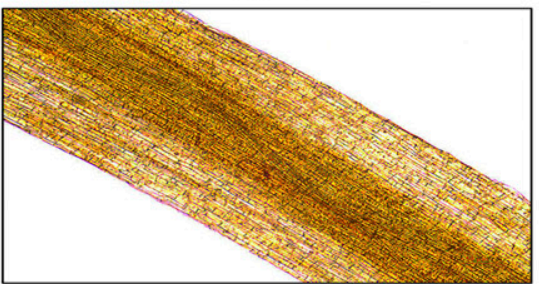

CK
B
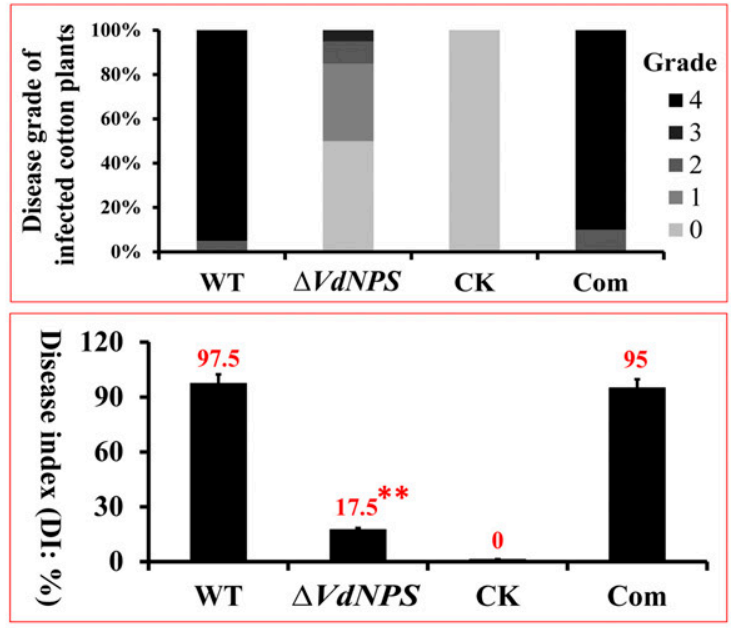

D 1

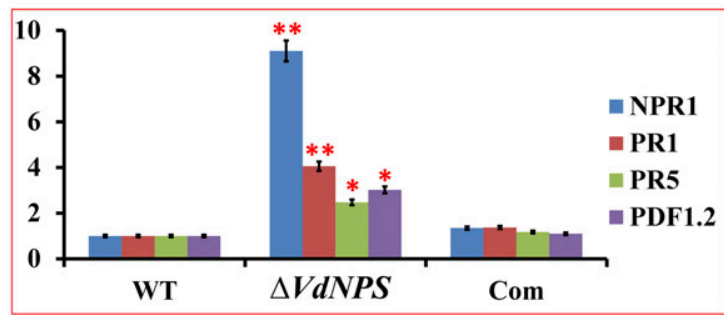

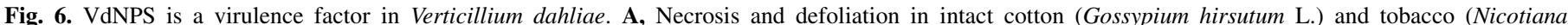

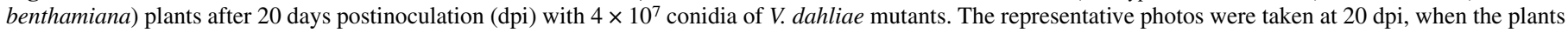

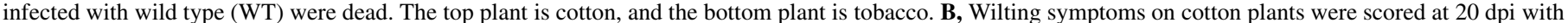

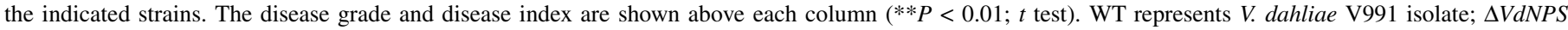

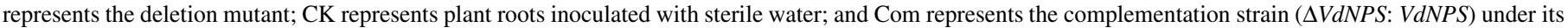

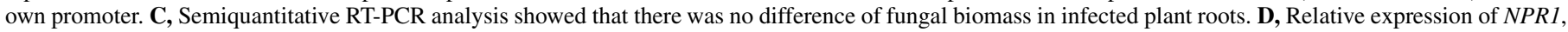

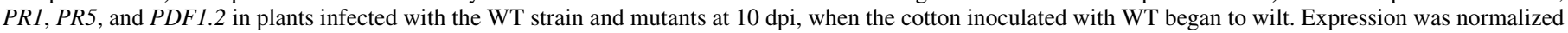

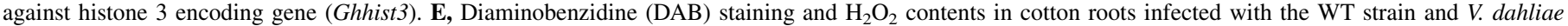
mutants. FW, fresh weight. Scale bar: $50 \mu \mathrm{m}$. Asterisks indicate significant differences $(* P<0.05 ; * * P<0.01 ; t$ test) between each mutant and WT. 
whereas DEGs encoding MYC2 were down-regulated (Fig. 7B and F); at 9 dpi, DEGs involved in JA-mediated signaling were down-regulated in $\triangle V d N P S$ (Fig. 7C and F). At 7 and 9 dpi, the gene expression in SAmediated signaling pathway was not different between WT and $\triangle V d N P S$. Besides the classical JA- and SA-mediated defense response, ET, auxin, ABA, gibberellin, cytokinin, and brassinosteroid-mediated signaling pathways were involved in the response to pathogen invasion, which were summarized in Supplementary Figures S9 and S10. In the process of plant-pathogen interaction (ko04626), $\mathrm{Ca}^{2+}$-mediated signaling pathways play an important role in $\triangle V d N P S$; however, fungal pathogen-associated molecular pattern (PAMP, Avr9) triggering immunity only occurred at 9 dpi (Fig. 7D and F). At 5 and 9 dpi, DEGs encoding calcium binding protein, calmodulin-like protein, and calcium-dependent protein kinase were all down-regulated in $\triangle V d N P S$; at $9 \mathrm{dpi}$, DEGs encoding the cyclic nucleotide-gated ion channel were up-regulated in $\triangle V d N P S$, whereas the plant receptor kinase BAK1 encoding gene was down-regulated (Supplementary Fig. S11). WRKY transcription factors 22 and 33 play an important role in disease resistance even though they are antagonistic (Supplementary Fig. S11). The above results indicated the enhanced plant hormone-mediated signal pathways, and the $\mathrm{Ca}^{2+}-\mathrm{WRKY}$ - and $\mathrm{Ca}^{2+}$-Rboh-mediated $\mathrm{HR}$, cell wall reinforcement and defense-related gene expressions induced by $\triangle V d N P S$ provided a likely explanation for the decreased virulence of $\triangle V d N P S$ mutants.

\section{DISCUSSION}

Since the discovery of penicillin, NPSs have been well characterized as important enzymes for synthesizing natural products in fungi and plant pathogens, including (i) $\beta$-lactam abiotic synthesis, such as penicillin and cephalosporin in Penicillium chrysogenum (Arnstein et al. 1959), Acremonium chrysogenum, and Streptomyces clavuligerus (Banko et al. 1986; Jensen et al. 1988); (ii) fungal toxins biosynthesis, such as HC-toxin in C. carbonum (Panaccione et al. 1992), AM-toxin in Alternaria alternata (Johnson et al. 2000), phytotoxin in Dickeya zeae (Cheng et al. 2013), and fusaoctaxin A in F. graminearum (Jia et al. 2019); and (iii) siderophore production in Alternaria alternata (Chen et al. 2013), C. heterostrophus, F. graminearum, and Alternaria brassicicola (Oide et al. 2006). VdNPS was first mentioned by Wang et al. (2018), who reported that as the homolog of key siderophore biosynthetic genes in Aspergillus nidulans (Eisendle et al. 2003),

Plant hormone signal transduction

A

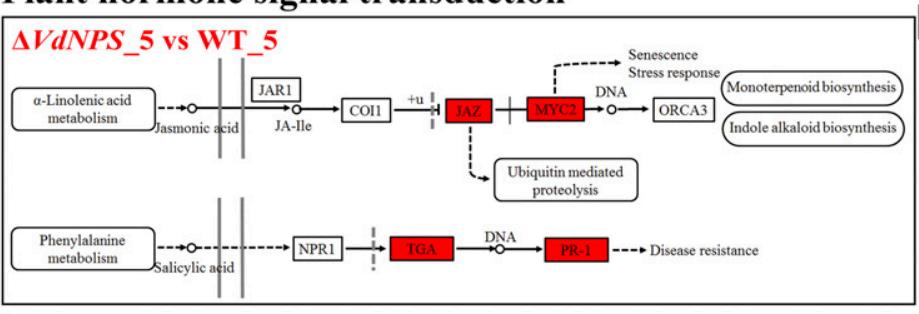

B

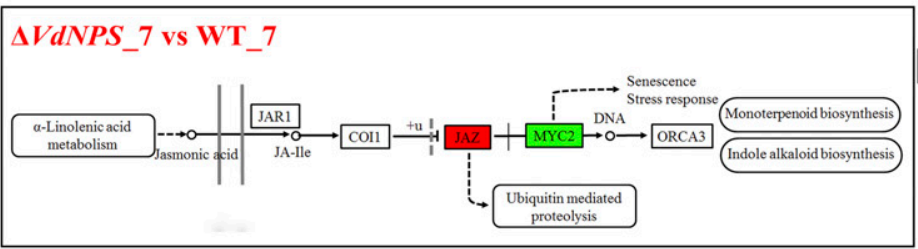

C

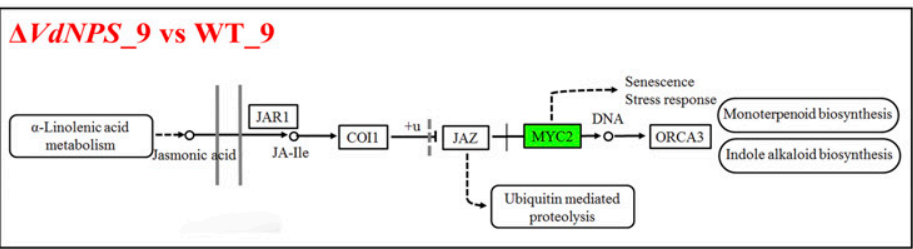

JAZ

$\mathbf{F}$

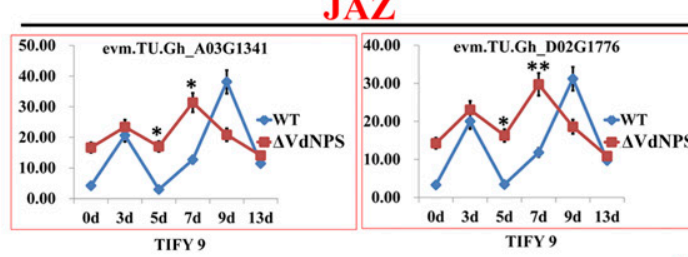

TGA
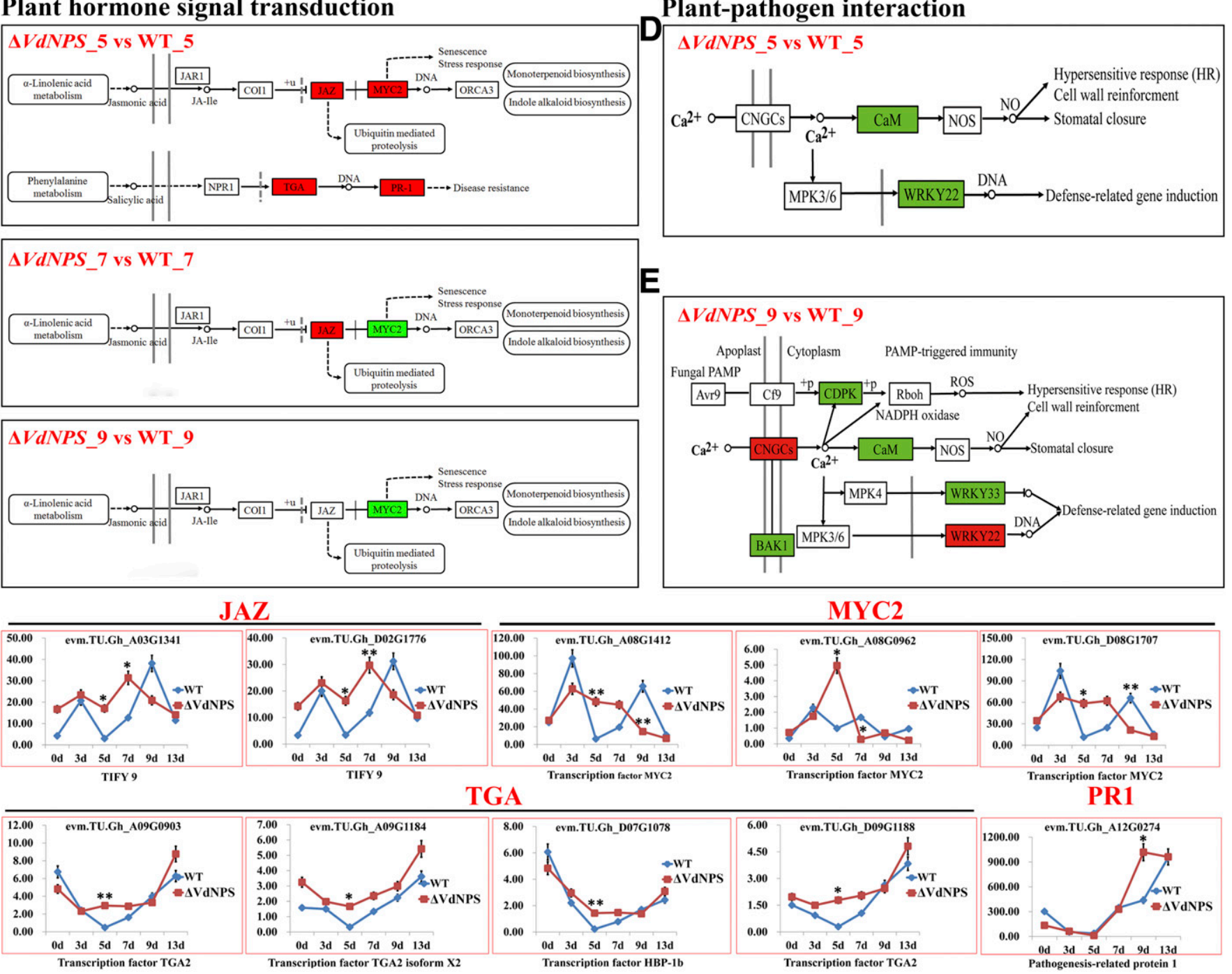

E

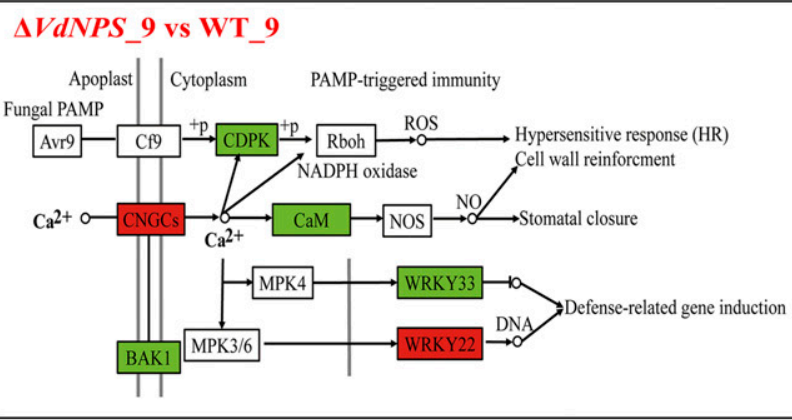

\section{MYC2}

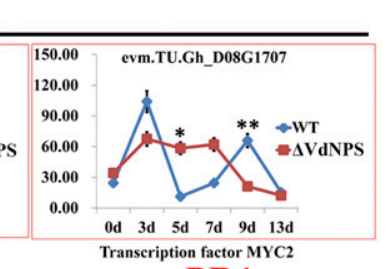

PR1

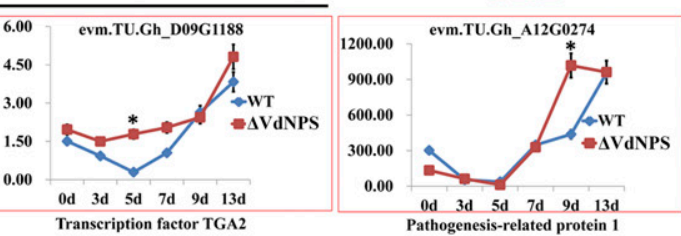

Fig. 7. Plant hormone signal transduction pathways and plant-pathogen interaction in cotton infected by wild type (WT) and $\triangle V d N P S$. A to C, After inoculating for 5, 7, and 9 days, differentially expressed genes (DEGs) in host were enriched in jasmonic acid (JA)- and salicylic acid (SA)-mediated signaling transduction pathways. At 5 dpi, DEGs involved in JA- and SA-mediated signaling pathways were all up-regulated. D and E, DEGs involved in plant-pathogen interaction. $\mathrm{Ca}^{2+}$-mediated host defense and fungal pathogen-associated molecular pattern (PAMP) triggered immunity are two predominantly different pathways in response to WT and $\triangle V d N P S$ infection. The red rectangles represent up-regulated DEGs, and the green rectangles represent down-regulated DEGs. F, The normalized expression of DEGs involved in the JA- and SA-mediated signaling transduction pathways. The $y$-axis represents the normalized expression, the $x$-axis represents the infection time. Error bars represent standard deviation. Asterisks indicate significant differences $(* P<0.05 ; * * P<0.01 ; * * * P<0.001 ; t$ test). 
NPS (VDAG_05314) was highly up-regulated under iron sufficiency compared with that under conditions of iron starvation in $V$. dahliae XS11 strain. However, no further functional characterization of $V d N P S$ was reported later. In this research, through HPLC-MS analysis, we found that the end product of VdNPS, L$\delta$-( $\alpha$-aminoadipoyl)-L-cysteinyl-D-valine (NRP), did not synthesize in the $\triangle V d N P S$ strain. Through phylogenetic analysis showed that the VdNPS was the ortholog of AaNPS6, which catalyzes the produce of coprogen siderophore, while the molecular weight and structure of VdNRP was different from that of siderophores. Further LC-MS profiling showed that no siderophore associated metabolites were significant differentially synthesized in $\triangle V d N P S$, while the metabolic synthesis of carbapenem antibiotics and toxin staurosporine were down-regulated in $\triangle V d N P S$ strain. Additionally, the phenotypes of $\triangle V d N P S$ and WT $V$. dahliae were not different under iron stress. All the results implied that VdNPS is more likely a synthetase for metabolites biosynthesis than siderophore production.

Ignoring whether the NPSs are involved in abiotic synthesis, toxins biosynthesis, or siderophore production, they are important for fungal pathogenicity in different pathogens, such as Magnaporthe oryzae (Bohnert 2004; Song et al. 2015), C. carbonum (Panaccione et al. 1992), and Aspergillus nidulans (Eisendle et al. 2003). Loss of NPS in these fungi resulted in reduced virulence on hosts. The same decreased virulence was also observed in $\triangle V d N P S$ strain. The deletion of $V d N P S$ led to reduced microsclerotial formation and lower conidiation in V. dahliae. However, contrary to NPS6 in Alternaria alternata, $C$. heterostrophus, $F$. graminearum, and Alternaria brassicicola (Chen et al. 2013, Oide et al. 2006), the deletion mutants $\triangle V d N P S$ were less sensitive to oxidative stress. The tolerance to $\mathrm{H}_{2} \mathrm{O}_{2}$ was mediated by siderophore bypass Fenton reaction in $C$. heterostrophus, $F$. graminearum, Alternaria alternata, and Alternaria brassicicola, in which the iron application could restore the tolerance of NPS6 deletion mutants to $\mathrm{H}_{2} \mathrm{O}_{2}$ (Chen et al. 2013; Oide et al. 2006). Intracellular ROS is important for the differentiation of appressoria-like structures, conidiation, and virulence in fungal pathogens (Oide et al. 2006; Chen et al. 2013); and ROS from pathogens are important for development and pathogenicity, which was confirmed by Nox family within filamentous fungi (Lara-Ortiz et al. 2003), such as in B. cinerea (Segmüller et al. 2008), M. oryzae (Egan et al. 2007), C. purpurea (Giesbert et al. 2008), Alternaria alternata (Hyon et al. 2010), and $V$. dahliae (Zhao et al. 2016). In V. dahliae, transcriptional profiling analysis indicated that compared with WT, the gene encoding NADPH oxidases (NoxB) was down-regulated under $\mathrm{H}_{2} \mathrm{O}_{2}$ stress in $\triangle V d N P S$, which could inhibit the appressoria formation, thereby decreased the pathogenicity. Additionally, genes associated with cell structure strengthen DNA repair, and $\mathrm{H}_{2} \mathrm{O}_{2}$ detoxification were up-regulated in $\triangle V d N P S$ under $\mathrm{H}_{2} \mathrm{O}_{2}$ stress. It maybe one factor for $\mathrm{H}_{2} \mathrm{O}_{2}$ tolerance in $\triangle V d N P S$. In Fenton reaction, transformation from ferrous $\left(\mathrm{Fe}^{2+}\right)$ to ferric $\left(\mathrm{Fe}^{3+}\right)$ is the key step to change the ROS to the highly active $\mathrm{OH}^{\bullet}$, which is the ROS damage form (Halliwell and Gutteridge 1992). Unexpectedly, as the genes encoding ferric associated transporters and reductase, and the genes encoding copper transporter and oxidase were differentially expressed, transcriptional profiling analysis indicated that besides iron, copper was involved in the response to $\mathrm{H}_{2} \mathrm{O}_{2}$ stress in $V$. dahliae. Under optimal level, copper induces cellular antioxidants to scavenge ROS thereby preventing the damage caused by the overproduction of ROS (Baker 1987). However, excessive copper will catalyze the ROS production via Haber Weiss and Fenton reactions (Aust et al. 1985). Without oxidative stress, iron and copper $(<30 \mu \mathrm{m})$ had no influence on the growth of WT and $\triangle V d N P S$ strain; while under $\mathrm{H}_{2} \mathrm{O}_{2}$ stress, the exogenous application of iron $(<10 \mu \mathrm{m})$ and copper $(<30 \mu \mathrm{m})$ could restore the tolerance to $\mathrm{H}_{2} \mathrm{O}_{2}$ in WT but had no obvious influence on $\triangle V d N P S$. The results indicated that the deletion of $V d N P S$ caused insensitivity to the iron and copper change, but it regulated ROS response via Fenton reaction.
The induction of PR gene expression, ROS burst, and SA/JA production are common defense responses of plants against pathogen infection (Fonseca et al. 2018). Our molecular demonstration and RNA-sequencing results indicated that $\triangle V d N P S$ induced most PR gene expression, higher ROS production, and stronger SA/JA-mediated signaling transduction. During the process of plant-pathogen interactions, fungi have evolved three strategies to interfere the host SA homeostasis and SA-mediated plant defenses (Chanclud and Morel 2016) as follows: (i) producing and secreting chorismate mutase as a virulence factor, such as $\mathrm{Cmu} 1$ in Ustilago maydis (Djamei et al. 2011); (ii) synthesizing secretory enzymes to degrade SA precursors to decrease SA levels in the plant host, such as isochorismatase in V. dahliae (Liu et al. 2014); and (iii) synthesizing salicylate hydroxylases to convert bioactive SA into an inactive compound, such as U. maydis (Rabe et al. 2013) and Epichloëfestucae (Ambrose et al. 2015). In this study, we found that $\triangle V d N P S$ was hypersensitive to SA stress. Further molecular characterization showed that the genes encoding salicylate hydroxylase was up-regulated in $\triangle V d N P S$ compared with WT, and the loss of these genes caused decreased virulence and inability to grow under SA stress. Researches have reported that SA could diffuse from cell to cell through membrane, thereby reducing the SA accumulation and maintain SA homeostasis (Shulaev et al. 1997). Base on the results, we speculated that during pathogen infection, the host SA synthesis was induced, the intracellular SA mediated the establishment of SAR (Durrant and Dong 2004), and the extracellular SA have direct antifungal activity. The salicylate hydroxylases in pathogens detoxify SA through SA hydroxylation and thereby promotes disease. It is the first report that $\triangle V d N P S$ induced stronger SA-mediated defense response and hypersensitivity to SA via up-regulation of salicylate hydroxylases. The relationship between VdNPS and VdSAH needs further demonstration.

In summary, VdNPS is a key virulent determinant in $V$. dahliae (Fig. 8). Uncovering the mechanism of actions of VdNPS should facilitate the development of cultivars with durable resistance against $V$. dahliae-associated diseases.

Data availability statement. The transcriptome data for this study were deposited into the NCBI database as a BioProject (accession numbers PRJNA587035 and PRJNA587036).

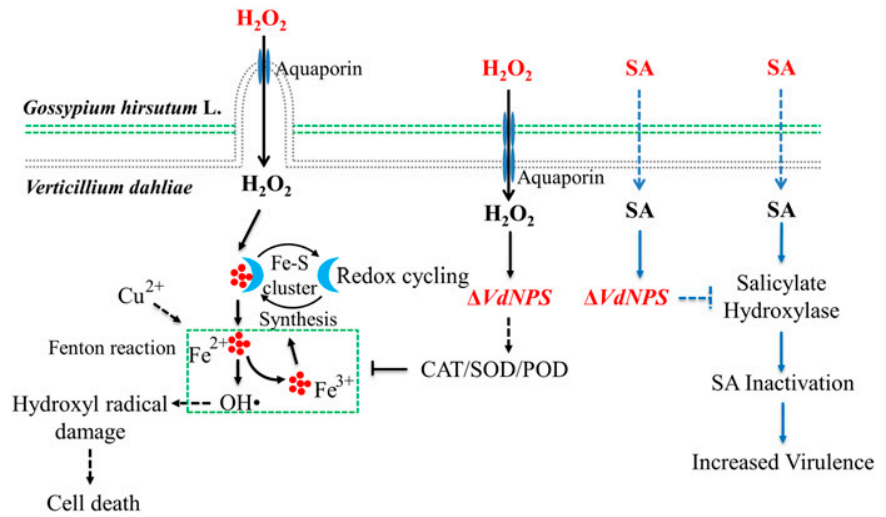

Fig. 8. A proposed working model of $V d N P S$ functions in oxidative and salicylic acid (SA) stress response. Under $\mathrm{H}_{2} \mathrm{O}_{2}$ stress, $\triangle V d N P S$ inhibited the transformation of $\mathrm{H}_{2} \mathrm{O}_{2}$ to $\mathrm{OH}^{\bullet}$ through up-regulating the expression of $V d C A T, V d S O D$, and $V d P O D$, and $\mathrm{Fe}^{2+} / \mathrm{Cu}^{2+}$-mediated Fenton reaction. Under SA stress, the genes associated SA hydroxylation were induced in wild-type Verticillium dahliae, thus stimulating the SA detoxification. However, the expression of genes encoding salicylate hydroxylases were down-regulated in $\triangle V d N P S$, and the $\triangle V d N P S$ mutants were hypersensitive to SA stress. Black lines indicate the response of $\triangle V d N P S$ to $\mathrm{H}_{2} \mathrm{O}_{2}$ stress. Gray/blue lines represent the response mechanism of $\triangle V d N P S$ to SA stress. Dotted lines represent that it's unsure whether the functional mechanism is direct or indirect. 


\section{LITERATURE CITED}

Alvarez, M. E. 2000. Salicylic acid in the machinery of hypersensitive cell death and disease resistance. Plant Mol. Biol. 44:429-442.

Ambrose, K. V., Tian, Z., Wang, Y., Smith, J., Zylstra, G., Huang, B., and Belanger, F. C. 2015. Functional characterization of salicylate hydroxylase from the fungal endophyte Epichloe festucae. Sci. Rep. 5:10939.

Apel, K., and Hirt, H. 2004. Reactive oxygen species: Metabolism, oxidative stress, and signal transduction. Annu. Rev. Plant Biol. 55:373-399.

Apostol, I., Heinstein, P. F., and Low, P. S. 1989. Rapid stimulation of an oxidative burst during elicitation of cultured plant cells role in defense and signal transduction. Plant Physiol. 90:109-116.

Arnstein, H. R., Morris, D., and Toms, E. J. 1959. Isolation of a tripeptide containing alpha-aminoadipic acid from the mycelium of Penicillium chrysogenum and its possible significance in penicillin biosynthesis. Biochim. Biophys. Acta 35:561-562.

Aust, S. D., Morehouse, L. A., and Thomas, C. E. 1985. Role of metals in oxygen radical reactions. J. Free Radic. Biol. Med. 1:3-25.

Baker, A. J. M. 1987. Metal tolerance. New Phytol. 106:93-111.

Banko, G., Wolfe, S., and Demain, A. L. 1986. Cell-free synthesis of delta-(1alpha-aminoadipyl)-1-cysteine, the frst intermediate of penicillin and cephalosporin biosynthesis. Biochem. Biophys. Res. Commun. 137: 528-535.

Bari, R., and Jones, J. D. G. 2009. Role of plant hormones in plant defence responses. Plant Mol. Biol. 69:473-488.

Bohnert, H. U. 2004. A putative polyketide synthase/peptide synthetase from Magnaporthe grisea signals pathogen attack to resistant rice. Plant Cell 16: 2499-2513.

Byford, M. F., Baldwin, J. E., Shiau, C. Y., and Schofield, C. J. 1997. The mechanism of ACV synthetase. Chem. Rev. 97:2631-2650.

Chanclud, E., and Morel, J. B. 2016. Plant hormones: A fungal point of view. Mol. Plant Pathol. 17:1289-1297.

Chen, L. H., Lin, C. H., and Chung, K. R. 2013. A nonribosomal peptide synthetase mediates siderophore production and virulence in the citrus fungal pathogen Alternaria alternata. Mol. Plant Pathol. 14:497-505.

Cheng, Y., Liu, X., An, S., Chang, C., Zou, Y., Huang, L., Zhong, J., Liu, Q., Jiang, Z., Zhou, J., and Zhang, L. H. 2013. A nonribosomal peptide synthase containing a stand-alone condensation domain is essential for phytotoxin zeamine biosynthesis. Mol. Plant-Microbe Interact. 26:1294-1301.

Daudi, A., Cheng, Z., O’Brien, J. A., Mammarella, N., Khan, S., Ausubel, F. M., and Bolwell, G. P. 2012. The apoplastic oxidative burst peroxidase in Arabidopsis is a major component of pattern-triggered immunity. Plant Cell 24:275-287.

Deng, W., Yongbo, W., Zexian, L., Han, C., and Yu, X. 2014. HemI: A toolkit for illustrating heatmaps. PLoS One 9:e111988.

Djamei, A., Schipper, K., Rabe, F., Ghosh, A., Vincon, V., Kahnt, J., Osorio, S., Tohge, T., Fernie, A. R., Feussner, I., Feussner, K., Meinicke, P., Stierhof, Y. D., Schwarz, H., Macek, B., Mann, M., and Kahmann, R. 2011. Metabolic priming by a secreted fungal effector. Nature 478:395-398.

Doke, N., Miura, Y., Sanchez, L., Park, H., Noritake, T., Yoshioka, H., and Kawakita, K. 1996. The oxidative burst protects plants against pathogen attack: Mechanism and role as an emergency signal for plant biodefence-a review. Gene 179:45-51.

Dong, X. 2004. NPR1, all things considered. Curr. Opin. Plant Biol. 7: 547-552.

Durrant, W., and Dong, X. 2004. Systemic acquired resistance. Annu. Rev. Phytopathol. 42:185-209.

Egan, M. J., Wang, Z., Jones, M. A., Smirnoff, N., and Talbot, N. J. 2007. Generation of reactive oxygen species by fungal NADPH oxidases is required for rice blast disease. Proc. Natl. Acad. Sci. USA 104:11772-11777.

Eisenberg, G. 1943. Colorimetric determination of hydrogen peroxide. Ind. Eng. Chem. Anal. Ed. 15:327-328.

Eisendle, M., Oberegger, H., Zadra, I., and Haas, H. 2003. The siderophore system is essential for viability of Aspergillus nidulans: Functional analysis of two genes encoding L-ornithine N5-monooxygenase (sidA) and a nonribosomal peptide synthetase (sidC). Mol. Microbiol. 49:359-375.

El-Gebali, S., Mistry, J., Bateman, A., Eddy, S. R., Luciani, A., Potter, S. C., Qureshi, M., Richardson, L. J., Salazar, G. A., Smart, A., Sonnhammer, E. L. L., Hirsh, L., Paladin, L., Piovesan, D., Tosatto, S. C. E., and Finn, R. D. 2018. The Pfam protein families database in 2019. Nucleic Acids Res. 47:D427-D432.

Fischbach, M. A., and Walsh, C. T. 2010. Assembly-line enzymology for polyketide and nonribosomal peptide antibiotics: Logic, machinery, and mechanisms. Chem. Rev. 37:3468-3496.

Fonseca, S., Radhakrishnan, D., Prasad, K., and Chini, A. 2018. Fungal production and manipulation of plant hormones. Curr. Med. Chem. 25:253-267.

Fradin, E. F., and Thomma, B. P. 2006. Physiology and molecular aspects of Verticillium wilt diseases caused by $V$. dahliae and $V$. albo-atrum. Mol. Plant Pathol. 7:71-86.
Fradin, E. F., Zhang, Z., Ayala, J. C. J., Castroverde, C. D. M., Nazar, R. N., Robb, J., Liu, C., and Thomma, B. P. H. J. 2009. Genetic dissection of Verticillium wilt resistance mediated by tomato Ve1. Plant Physiol. 150: 320-332.

Fraga, C. G., Clowers, B. H., Moore, R. J., and Zink, E. M. 2010. Signaturediscovery approach for sample matching of a nerve-agent precursor using liquid chromatography-mass spectrometry, XCMS, and chemometrics. Anal. Chem. 82:4165-4173.

Gao, W., Long, L., Zhu, L. F., Xu, L., Gao, W. H., Sun, L. Q., Liu, L. L., and Zhang, X. L. 2013a. Proteomic and virus-induced gene silencing (VIGS) analyses reveal that gossypol, brassinosteroids, and jasmonic acid contribute to the resistance of cotton to Verticillium dahliae. Mol. Cell. Proteomics $12: 3690-3703$

Gao, X., Li, F., Li, M., Kianinejad, A. S., Dever, J. K., Wheeler, T. A., Li, Z., He, P., and Shan, L. 2013b. Cotton GhBAK1 mediates Verticillium wilt resistance and cell death. J. Integr. Plant Biol. 55:586-596.

Gao, X., Wheeler, T., Li, Z., Kenerley, C. M., He, P., and Shan, L. 2011. Silencing GhNDR1 and GhMKK2 compromises cotton resistance to Verticillium wilt. Plant J. 66:293-305.

Giesbert, S., Schuerg, T., Scheele, S., and Tudzynski, P. 2008. The NADPH oxidase Cpnox 1 is required for full pathogenicity of the ergot fungus Claviceps purpurea. Mol. Plant Pathol. 9:317-327.

Gkizi, D., Lehmann, S., L'Haridon, F., Serrano, M., Paplomatas, E. J., Métraux, J., and Tjamos, S. E. 2016. The innate immune signaling system as a regulator of disease resistance and induced systemic resistance activity against Verticillium dahliae. Mol. Plant-Microbe Interact. 29: 313-323.

Gómez, L., Felix, G., and Boller, T. 1999. A single locus determines sensitivity to bacterial flagellin in Arabidopsis thaliana. Plant J. 18:277-284.

Grant, M., and Lamb, C. 2006. Systemic immunity. Curr. Opin. Plant Biol. 9: 414-420.

Grant, M. R., and Jones, J. D. 2009. Hormone (dis) harmony moulds plant health and disease. Science 324:750-752.

Guo, M., Chen, Y., Du, Y., Dong, Y., Guo, W., Zhai, S., Zhang, H., Dong, S., Zhang, Z., and Wang, Y. 2011. The bZIP transcription factor MoAP1 mediates the oxidative stress response and is critical for pathogenicity of the rice blast fungus Magnaporthe oryzae. PLoS Pathog 7:e1001302.

Gupta, N., Limbago, B. M., Patel, J. B., and Kallen, A. J. 2011. Carbapenemresistant Enterobacteriaceae: Epidemiology and prevention. Clin. Infect. Dis. 53:60-67.

Haese, A., Schubert, M., Herrmann, M., and Zocher, R. 1993. Molecular characterization of the enniatin synthetase gene encoding a multifunctional enzyme catalyzing $N$-methyldepsipeptide formation in Fusarium scirpi. Mol. Microbiol. 7:905-914.

Halliwell, B., and Gutteridge, J. M. 1992. Biologically relevant metal iondependent hydroxyl radical generation. An update. FEBS Lett. 307: 108-112.

Hwang, I. S., and Hwang, B. K. 2011. The pepper mannose-binding lectin gene $C a M B L 1$ is required to regulate cell death and defense responses to microbial pathogens. Plant Physiol. 155:447-463.

Hyon, G. S., Ikeda, K., Hosogi, N., Shinogi, T., and Park, P. 2010. Inhibitory effects of antioxidant reagent in reactive oxygen species generation and penetration of appressoria of Alternaria alternata Japanese pear pathotype. Phytopathology 100:840-847.

Jacobsen, M. D., Weil, M., and Raff, M. C. 1996. Role of Ced-3/ICE-family proteases in staurosporine-induced programmed cell death. J. Cell Biol. 133:1041-1051.

Jensen, S. E., Westlake, D. W. S., and Wolfe, S. 1988. Production of the penicillin precursor $\delta$-(l- $\alpha$-aminoadipyl)-l-cysteinyl-d-valine (ACV) by cell-free extracts from Streptomyces clavuligerus. FEMS Microbiol. Lett. 49:213-218

Jia, L. J., Tang, H. Y., Wang, W. Q., Yuan, T. L., Wei, W. Q., Pang, B., Gong, X. M., Wang, S. F., Li, Y. J., Zhang, D., Liu, W., and Tang, W. H. 2019. A linear nonribosomal octapeptide from Fusarium graminearum facilitates cell-to-cell invasion of wheat. Nat. Commun. 10:922

Johnson, R. D., Johnson, L., Itoh, Y., Kodama, M., Otani, H., and Kahmoto, K. 2000. Cloning and characterization of a cyclic peptide synthetase gene from Alternaria alternata apple pathotype whose product is involved in AM-toxin synthesis and pathogenicity. Mol. Plant-Microbe Interact. 13: 742-753.

Kim, D. S., and Hwang, B. K. 2011. The pepper receptor-like cytoplasmic protein kinase CaPIK1 is involved in plant signaling of defense and celldeath responses. Plant J. 66:642-655.

Klosterman, S. J., Subbarao, K. V., Kang, S., Veronese, P., Gold, S. E., Thomma, B. P., Chen, Z., Henrissat, B., Lee, Y. H., and Park, J. 2011. Comparative genomics yields insights into niche adaptation of plant vascular wilt pathogens. PLoS Pathog 7:e1002137.

Kohanski, M. A., Dwyer, D. J., and Collins, J. J. 2010. How antibiotics kill bacteria: From targets to networks. Nat. Rev. Microbiol. 8:423-435. 
Kohanski, M. A., Dwyer, D. J., Hayete, B., Lawrence, C. A., and Collins, J. J. 2007. A common mechanism of cellular death induced by bactericidal antibiotics. Cell 130:797-810.

Kovtun, Y., Chiu, W., Tena, G., and Sheen, J. 2000. Functional analysis of oxidative stress-activated mitogen-activated protein kinase cascade in plants. Proc. Natl. Acad. Sci. 97:2940-2945.

Krishnamurthy, A., and Rathinasabapathi, B. 2013. Oxidative stress tolerance in plants: Novel interplay between auxin and reactive oxygen species signaling. Plant Signal. Behav. 8:e25761.

Lara-Ortiz, T., Riveros-Rosas, H., and Aguirre, J. 2003. Reactive oxygen species generated by microbial NADPH oxidase NoxA regulate sexual development in Aspergillus nidulans. Mol. Microbiol. 50:1241-1255.

Li, C., He, X., Luo, X., Xu, L., Liu, L., Min, L., Jin, L., Zhu, L., and Zhang, X. 2014. Cotton WRKY1 mediates the plant defense-to-development transition during infection of cotton by Verticillium dahliae by activating JASMONATE ZIM-DOMAIN1 expression. Plant Physiol. 166:2179-2194.

Li, Y., Han, L., Wang, H., Zhang, J., Sun, S., Feng, D., Yang, C., Sun, Y., Zhong, N., and Xia, G. 2016. The thioredoxin GbNRX1 plays a crucial role in homeostasis of apoplastic reactive oxygen species in response to Verticillium dahliae infection in cotton. Plant Physiol. 170:2392-2406.

Liu, Y., and Chen, Y. 2007. High-efficiency thermal asymmetric interlaced PCR for amplification of unknown flanking sequences. Biotechniques 43: 649-656.

Liu, T., Song, T., Zhang, X., Yuan, H., Su, L., Li, W., Xu, J., Liu, S., Chen, L., and Chen, T. 2014. Unconventionally secreted effectors of two filamentous pathogens target plant salicylate biosynthesis. Nat. Commun. 5:4686.

Lorrain, S., Vailleau, F., Balagué, C., and Roby, D. 2003. Lesion mimic mutants: Keys for deciphering cell death and defense pathways in plants? Trends Plant Sci. 8:263-271.

Luo, P., Wang, Y., Wang, G., Essenberg, M., and Chen, X. 2001. Molecular cloning and functional identification of $(+)-\delta$-cadinene-8-hydroxylase, a cytochrome P450 mono-oxygenase (CYP706B1) of cotton sesquiterpene biosynthesis. Plant J. 28:95-104.

Luo, X., Mao, H., Wei, Y., Cai, J., Xie, C., Sui, A., Yang, X., and Dong, J. 2016. The fungal-specific transcription factor Vdpf influences conidia production, melanized microsclerotia formation and pathogenicity in Verticillium dahliae. Mol. Plant Pathol. 17:1364-1381.

Luo, X., Xie, C., Dong, J., and Yang, X. 2019. Comparative transcriptome analysis reveals regulatory networks and key genes of microsclerotia formation in the cotton vascular wilt pathogen. Fungal Genet. Biol. 126:25-36.

Luo, X., Xie, C., Dong, J., Yang, X., and Sui, A. 2014. Interactions between Verticillium dahliae and its host: Vegetative growth, pathogenicity, plant immunity. Appl. Microbiol. Biotechnol. 98:6921-6932.

Marahiel, M. A. 2016. A structural model for multimodular NRPS assembly lines. Nat. Prod. Rep. 33:136-140.

Mou, Z., Fan, W., and Dong, X. 2003. Inducers of plant systemic acquired resistance regulate NPR1 function through redox changes. Cell 113: 935-944.

Oide, S., Moeder, W., Krasnoff, S., Gibson, D., Haas, H., Yoshioka, K., and Turgeon, B. G. 2006. NPS6, encoding a nonribosomal peptide synthetase involved in siderophore-mediated iron metabolism, is a conserved virulence determinant of plant pathogenic ascomycetes. Plant Cell 18:2836-2853.

Panaccione, D. G., Scottcraig, J. S., Pocard, J. A., and Walton, J. D. 1992. A cyclic peptide synthetase gene required for pathogenicity of the fungus Cochliobolus carbonum on maize. Proc. Natl. Acad. Sci. USA 89: 6590-6594.

Pegg, G. F., and Brady, B. L. 2002. Verticillium Wilts. CABI, Wallingford, U.K.

Puertollano, M. A., Puertollano, E., Alvarez de Cienfuegos, G., and de Pablo, M. A. 2011. Dietary antioxidants: Immunity and host defense. Curr. Top. Med. Chem. 11:1752-1766

Qin, J., Zhao, J., Zuo, K., Cao, Y., Ling, H., Sun, X., and Tang, K. 2004. Isolation and characterization of an ERF-like gene from Gossypium barbadense. Plant Sci. 167:1383-1389.

Rabe, F., Ajami-Rashidi, Z., Doehlemann, G., Kahmann, R., and Djamei, A. 2013. Degradation of the plant defence hormone salicylic acid by the biotrophic fungus Ustilago maydis. Mol. Microbiol. 89:179-188.

Satterfield, C. N., and Bonnell, A. H. 1955. Interferences in titanium sulfate method for hydrogen peroxide. Anal. Chem. 27:1174-1175.

Segmüller, N., Kokkelink, L., Giesbert, S., Odinius, D., van Kan, J., and Tudzynski, P. 2008. NADPH Oxidases are involved in differentiation and pathogenicity in Botrytis cinerea. Mol. Plant-Microbe Interact. 21:808-819.
Seo, S., Seto, H., Yamakawa, H., and Ohashi, Y. 2001. Transient accumulation of jasmonic acid during the synchronized hypersensitive cell death in tobacco mosaic virus-infected tobacco leaves. Mol. Plant-Microbe Interact. 14:261-264

Shirasu, K., Nakajima, H., Rajasekhar, V. K., Dixon, R. A., and Lamb, C. 1997. Salicylic acid potentiates an agonist-dependent gain control that amplifies pathogen signals in the activation of defense mechanisms. Plant Cell 9:261-270.

Shulaev, V., Silverman, P., and Raskin, I. 1997. Airborne signaling by methyl salicylate in plant pathogen resistance. Nature 385:718-721.

Sieber, S. A., and Marahiel, M. A. 2005. Molecular mechanisms underlying nonribosomal peptide synthesis: approaches to new antibiotics. ChemInform 36:715-738.

Song, Z., Bakeer, W., Marshall, J. W., Ahmed, A., Yakasai, A. A., Khalid, R. M., Collemare, J., Skellam, E., Tharreau, D., Lebrun, M. H., Lazarus, C. M., Bailey, A. M., Simpson, T. J., and Cox, R. J. 2015. Heterologous expression of the avirulence gene ACE1 from the fungal rice pathogen Magnaporthe oryzae. Chem. Sci. (Camb.) 6:4837-4845.

Sun, L., Zhu, L., Xu, L., Yuan, D., Min, L., and Zhang, X. 2014. Cotton cytochrome P450 CYP82D regulates systemic cell death by modulating the octadecanoid pathway. Nat. Commun. 5:5372.

Tan, X. P., Liang, W. Q., Liu, C. J., Luo, P., Heinstein, P., and Chen, X. Y. 2000. Expression pattern of $(+)-\delta$-cadinene synthase genes and biosynthesis of sesquiterpene aldehydes in plants of Gossypium arboreum L. Planta 210: 644-651.

Tarry, M. J., and Schmeing, T. M. 2015. Specific disulfide cross-linking to constrict the mobile carrier domain of nonribosomal peptide synthetases. Protein Eng. Des. Selection Pediatr. 28:163-170.

Tamaoki, T., Hisayo, N., Isami, T., Yuzuru, K., and Fusao, T. 1986. Staurosporine, a potent inhibitor of phospholipid/ $\mathrm{Ca}^{++}$dependent protein kinase. Biochem. Biophys. Res. Commun. 135:397-402.

Ton, J., Van Pelt, J. A., van Loon, L. C., and Pieterse, C. M. 2002. Differential effectiveness of salicylate-dependent and jasmonate/ethylene-dependent induced resistance in Arabidopsis. Mol. Plant-Microbe Interact. 15: 27-34.

Torres, M. A. 2010. ROS in biotic interactions. Physiol. Plant. 138:414-429.

Torres, M. A., Jones, J. D., and Dangl, J. L. 2006. Reactive oxygen species signaling in response to pathogens. Plant Physiol. 141:373-378.

van Loon, L., Rep, M., and Pieterse, C. 2006. Significance of inducible defense-related proteins in infected plants. Annu. Rev. Phytopathol. 44: 135-162.

Vellosillo, T., Vicente, J., Kulasekaran, S., Hamberg, M., and Castresana, C. 2010. Emerging complexity in reactive oxygen species production and signaling during the response of plants to pathogens. Plant Physiol. 154: 444-448

Wang, Y. L., Deng, C. L., Tian, L., Xiong, D., Tian, C., and Klosterman, S. J. 2018. The transcription factor Vdhapx controls iron homeostasis and is crucial for virulence in the vascular pathogen Verticillium dahliae. MSphere 3:e00400-18.

Xu, L., Zhu, L., Tu, L., Liu, L., Yuan, D., Jin, L., Long, L., and Zhang, X. 2011. Lignin metabolism has a central role in the resistance of cotton to the wilt fungus Verticillium dahliae as revealed by RNA-Seq-dependent transcriptional analysis and histochemistry. J. Exp. Bot. 62:5607-5621.

Yang, C. L., Liang, S., Wang, H. Y., Han, L. B., Wang, F. X., Cheng, H. Q., Wu, X. M., Qu, Z. L., Wu, J. H., and Xia, G. X. 2015. Cotton major latex protein 28 functions as a positive regulator of the ethylene responsive factor 6 in defense against Verticillium dahliae. Mol. Plant 8:399-411.

Yoshioka, H., Numata, N., Nakajima, K., Katou, S., Kawakita, K., Rowland, O., Jones, J. D., and Doke, N. 2003. Nicotiana benthamiana gp91phox homologs NbrbohA and NbrbohB participate in $\mathrm{H}_{2} \mathrm{O}_{2}$ accumulation and resistance to Phytophthora infestans. Plant Cell 15:706-718.

Yu, J., Sook, J., Chun-Huai, C., Ficklin, S. P., Taein, L., Ping, Z., Don, J., Percy, R. G., and Dorrie, M. 2014. CottonGen: A genomics, genetics and breeding database for cotton research. Nucleic Acids Res.: 42:D1229D1236.

Zhang, Y., Zhao, L., Zhao, J., Li, Y., Wang, J., Guo, R., Gan, S. S., Liu, C. J., and Zhang, K. 2017. S5H/DMR6 encodes a salicylic acid 5-hydroxylase that fine-tunes salicylic acid homeostasis. Plant Physiol. 175:1082-1093.

Zhao, Y. L., Zhou, T. T., and Guo, H. S. 2016. Hyphopodium-specific VdNoxB/VdPls1-dependent ROS-Ca ${ }^{2+}$ signaling is required for plant infection by Verticillium dahliae. PLoS Pathog12:e1005793. 